\title{
HIGH ORDER TRANSMISSION CONDITIONS FOR THIN CONDUCTIVE SHEETS IN MAGNETO-QUASISTATICS
}

\author{
Kersten Schmidt ${ }^{1}$ And SÉBAstien TORdeuX ${ }^{2}$
}

\begin{abstract}
We propose transmission conditions of order 1,2 and 3 approximating the shielding behaviour of thin conducting curved sheets for the magneto-quasistatic eddy current model in 2D. This model reduction applies to sheets whose thicknesses $\varepsilon$ are at the order of the skin depth or essentially smaller. The sheet has itself not to be resolved, only its midline is represented by an interface. The computation is directly in one step with almost no additional cost. We prove the well-posedness w.r.t. to the small parameter $\varepsilon$ and obtain optimal bound for the modelling error outside the sheet of order $\varepsilon^{N+1}$ for the condition of order $N$. We end the paper with numerical experiments involving high order finite elements for sheets with varying curvature.
\end{abstract}

Mathematics Subject Classification. 65N30, 35C20, 35J25, 41A60, 35B40, 78M30, 78M35.

Received April 17, 2010. Revised February 16, 2011.

Published online June 28, 2011

\section{INTRODUCTION}

\subsection{Bibliographic overview}

In many practical applications, electronic devices are surrounded by casings or other sheets of a highly conductive material to protect them from external electromagnetic fields (e.g., protection of the signal in data cables) or to protect the environment from the electromagnetic fields generated by devices (e.g., transformer or bushings). To minimise the cost, size and weight, these sheets have to be thin. This leads to a non-perfect shielding where the electromagnetic fields partly penetrate the shields and have a small but significant effect in the protected region. The large ratio of characteristic lengths (width of the device against thickness of the sheet) leads to serious numerical problems. Indeed the classical numerical methods such as finite differences or finite elements require a small mesh size and are consequently very costly or simply not able (due to limited memory) to compute a numerical approximation of the solution of such a problem. Another important issue for such problems is related to mesh generation. It is time consuming to take into account small details in the geometry. Moreover, most of commercial mesh generators generate meshes with poor quality when the geometrical characteristic lengths are too different. Designing methods where the sheet needs not be included

\footnotetext{
Keywords and phrases. Asymptotic expansions, transmission condition, thin conducting sheets.

${ }^{1}$ Seminar for Applied Mathematics, ETH Zurich, 8092 Zurich, Switzerland, Project POEMS, INRIA Paris-Rocquencourt, 78153 Le Chesnay, France, currently at TU Berlin and DFG Research center MATHeon, 10623 Berlin, Germany. kersten.schmidt@math.tu-berlin.de

${ }^{2}$ Laboratoire de Mathématiques et de leurs Applications, UMR 5142, Université de Pau et des Pays de l'Adour, 64013 Pau, France, Project MAGIQUE-3D, INRIA Bordeaux-Sud-Ouest, 64013 Pau, France. sebastien.tordeux@univ-pau.fr
} 
in the mesh is really a crucial objective. These two considerations point out the necessity of an appropriate modelling of the shielding behaviour by thin sheets. This is the problem that we address in this paper.

Impedance boundary conditions (IBC), have been originally proposed for solid conductors by Shchukin [27] and Leontovich [17], for which recently IBCs of higher orders $[1,10,12,26]$ as for perfect conductors with thin coatings $[2,3,8]$ located at the boundary of the physical domain have been derived.

For thin layers located in the interior of the domain, approximate transmission conditions (also called impedance boundary conditions) of low order have been proposed by several authors, e.g., [14,16,20], and also for transient analysis [19]. Asymptotic expansions to any order have been derived for the electro-quasistatic equations [22] and time-harmonic Maxwell equations [21] in biological cells with isolating membranes.

In a previous article [24] we derived an asymptotic expansion at any order for the eddy current problem in 2D with thin highly conducting sheets. These derivations lead to the definition of a limit solution and correctors of higher orders, which can be computed iteratively. However, this multi-step procedure is not perfectly suited for the applications. In this article, we derive and study a one step numerical method to predict the shielding of a thin sheet.

\subsection{The geometrical setting}

Let us denote by $\vec{x}=(x, y, z)$ a cartesian parametrisation of $\mathbb{R}^{3}$ and by $\overrightarrow{\mathrm{e}_{x}}, \overrightarrow{\mathrm{e}_{y}}$ and $\overrightarrow{\mathrm{e}_{z}}$ the associated orthogonal unit vectors. To avoid difficulties mostly related to differential geometry, we will be concerned in this article with a $z$-invariant configuration. To take care of the two-dimensional phenomenon, we introduce the vector $\underline{x}=(x, y)$ composed of the two first coordinates of $\vec{x}$.

The computational domain $\Omega \times \mathbb{R}$ is decomposed into a highly conducting sheet $\Omega_{\text {int }}^{\varepsilon} \times \mathbb{R}$ and a domain $\Omega_{\text {ext }}^{\varepsilon} \times \mathbb{R}$ filled with air which satisfies

(i) $\Omega$ is a connected bounded domain of $\mathbb{R}^{2}$ with regular boundary $\partial \Omega$.

(ii) The conducting sheet $\Omega_{\text {int }}^{\varepsilon}$ is filled with material of constant conductivity $\sigma$ and permeability $\mu_{0}$. It has constant thickness $\varepsilon>0$ and is centred around $\Gamma$ a regular closed curve of $\Omega$ with no cross point

$$
\Omega_{\mathrm{int}}^{\varepsilon}=\left\{\underline{x} \in \Omega: \exists y \in \Gamma \quad\|\underline{x}-\underline{y}\|_{2}<\frac{\varepsilon}{2}\right\} .
$$

To each point of $\Gamma$ can be associated a curvature $\kappa(t)$ and a left normal unit vector $\underline{n}(t)$ (see Fig. 1$)$. Moreover it can be parameterised, for $\varepsilon$ small enough, by a local coordinate system as follows. Let $\hat{\Gamma}$ be a one-dimensional torus of the same length than $\Gamma$. Let denote by $\underline{x}_{m}: \hat{\Gamma} \rightarrow \Omega$ an injective $C^{\infty}$ mapping whose range is $\Gamma$ and satisfying $\left\|\underline{x}_{m}^{\prime}\right\|=1$. The domain $\Omega_{\text {int }}^{\varepsilon}$ can then be seen as the range of the bijective mapping

$$
\left\{\begin{array}{ccc}
\hat{\Gamma} \times]-\varepsilon / 2, \varepsilon / 2[ & \longrightarrow & \Omega_{\mathrm{int}}^{\varepsilon} \\
(t, s) & \longmapsto \underline{x}(t, s)=\underline{x}_{m}(t)+s \underline{n}(t) .
\end{array}\right.
$$

To obtain a $C^{\infty}$ sheet $\Omega_{\text {int }}^{\varepsilon}$ we assume that $\varepsilon\|\kappa\|_{L^{\infty}(\Gamma)}<2$.

(iii) The exterior of the sheet $\Omega_{\text {ext }}^{\varepsilon}=\Omega \backslash \overline{\Omega_{\text {int }}^{\varepsilon}}$ has constant permeability $\mu_{0}$ and is not conductive.

\subsection{Two-dimensional magneto-quasistatic with eddy current modelling}

When the geometric characteristic length are all much smaller than the wave length, the electromagnetic fields are accurately described by the eddy current model, a quasi-static approximation to the Maxwell equations [5,25],

$$
\left\{\begin{array}{l}
\operatorname{div}(\vec{E})=0 \\
\overrightarrow{\operatorname{rot}}(\vec{E})=-\partial_{t} \vec{B} \\
\overrightarrow{\operatorname{rot}}(\vec{B})=\mu_{0} \vec{J}
\end{array}\right.
$$




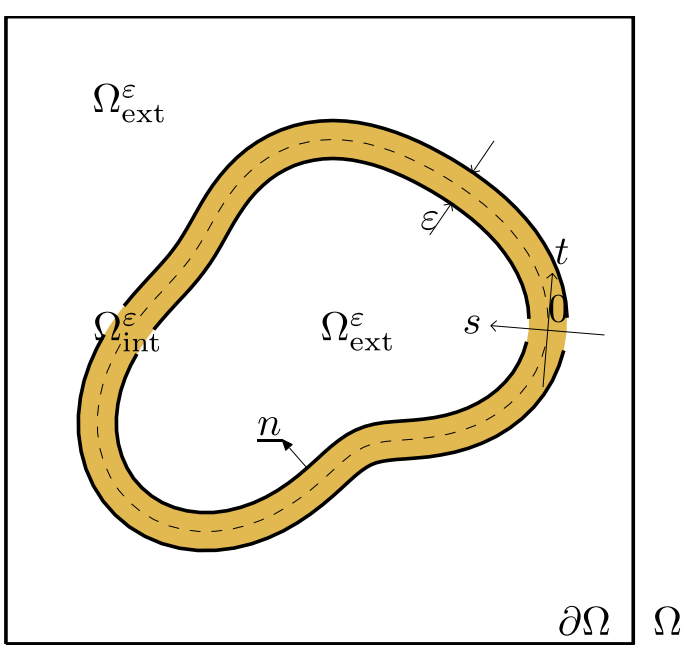

(a) Illustration of the geometry and the local coordinate system inside the sheet.

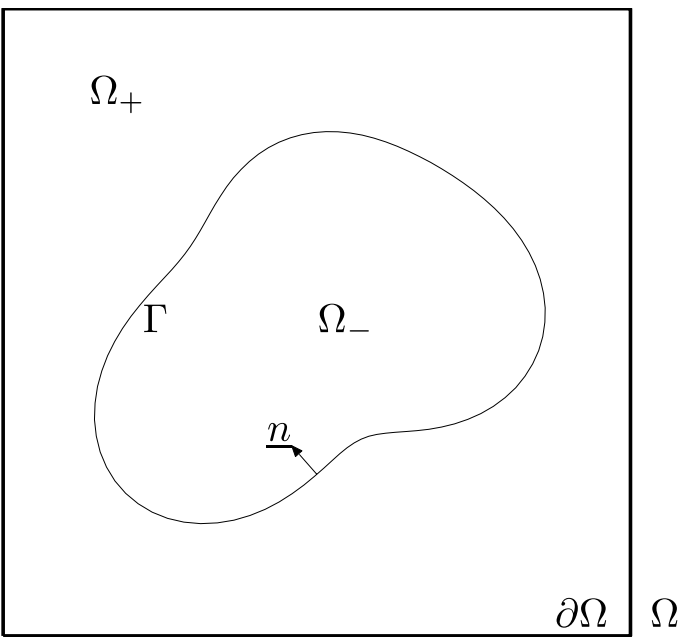

(b) The limit geometry of $\Omega_{\text {ext }}^{\varepsilon}$ is the whole domain without the midline $\Gamma$ of the sheet which consists of two connected parts $\Omega_{+}$and $\Omega_{-}$.

Figure 1. The two-dimensional geometrical setting for a sheet of thickness $\varepsilon$ and the limit geometry for $\varepsilon \rightarrow 0$.

where the current $\vec{J}$ is

- in $\Omega_{\mathrm{ext}}^{\varepsilon}$ impressed

$$
\vec{J}=\overrightarrow{J_{0}}
$$

with $\overrightarrow{J_{0}}$ a given regular function compactly supported in $\Omega_{\text {ext }}^{\varepsilon}$ (see Fig. 1b).

- in $\Omega_{\text {int }}^{\varepsilon}$ proportional to the electric field $\vec{E}$ by Ohms law

$$
\vec{J}=\sigma \vec{E} .
$$

We consider a time-harmonic excitation

$$
\overrightarrow{J_{0}}(\vec{x}, t)=\exp (-\mathrm{i} \omega t) j_{0}(\underline{x}) \overrightarrow{\mathrm{e}}_{z} .
$$

Due to $z$-invariance, the electromagnetic fields have to be sought in frequency domain with the form

$$
\vec{E}(\vec{x}, t)=e(\underline{x}) \exp (-\mathrm{i} \omega t) \overrightarrow{\mathrm{e}}_{z} \quad \text { and } \quad \vec{B}(\vec{x}, t)=b_{x}(\underline{x}) \exp (-\mathrm{i} \omega t) \overrightarrow{\mathrm{e}}_{x}+b_{y}(\underline{x}) \exp (-\mathrm{i} \omega t) \overrightarrow{\mathrm{e}}_{y} .
$$

Inside $\Omega_{\mathrm{int}}^{\varepsilon}$ and $\Omega_{\mathrm{ext}}^{\varepsilon}$ it reads for the out-of-plane electric field [23]

$$
\begin{aligned}
-\Delta e(\underline{x}) & =-\mathrm{i} \omega \mu_{0} j_{0}(\underline{x})=: f(\underline{x}), & & \text { in } \Omega_{\mathrm{ext}}^{\varepsilon}, \\
-\Delta e(\underline{x})+\mathrm{i} \omega \mu_{0} \sigma e(\underline{x}) & =0, & & \text { in } \Omega_{\mathrm{int}}^{\varepsilon} .
\end{aligned}
$$

with the perfectly conducting boundary condition on $\partial \Omega, e=0$, and transmission conditions at the two interfaces between $\Omega_{\mathrm{int}}^{\varepsilon}$ and $\Omega_{\mathrm{ext}}^{\varepsilon}$ : the function $e$ and its normal derivative are continuous across the interfaces or equivalently

$$
e \in H_{\Delta}^{1}(\Omega):=\left\{u \in H^{1}(\Omega): \Delta u \in L^{2}(\Omega) \text { and } u=0 \text { on } \partial \Omega\right\} .
$$


Finally, to express that the sheet is highly conductive, we rescale the coefficient $i \omega \mu_{0} \sigma$ by multiplying it by the small thickness $\varepsilon$. Thus, we introduce a quantity

$$
c_{0}=\varepsilon \omega \mu_{0} \sigma
$$

To obtain asymptotic solutions for small values of the thickness $\varepsilon$ we will let it vary, while defining $c_{0}$ by $(1.11)$ for some fixed thickness $\varepsilon_{0}$ and so independently of $\varepsilon$. Concurrently, we use the notation $e^{\varepsilon}$ for the electric field solution highlighting that it depends on the varying $\varepsilon$.

We have now all the ingredients to set a system of PDEs defining the exact solution. The problem we investigate is to find the electrical field $e^{\varepsilon}$ satisfying

$$
\begin{cases}e^{\varepsilon} \in H_{\Delta}^{1}(\Omega), & \text { in } \Omega_{\mathrm{ext}}^{\varepsilon} \\ -\Delta e^{\varepsilon}(\underline{x})=f(\underline{x}), & \text { in } \Omega_{\mathrm{int}}^{\varepsilon} .\end{cases}
$$

Remark 1.1 (asymptotic solution with scaled coefficient $i \omega \mu_{0} \sigma$ ). Scaling $\omega \mu_{0} \sigma$ like $1 / \varepsilon$ does not have at first glance a clear meaning. Indeed a thin sheet has, in real configuration, a given thickness $(\varepsilon=1 \mathrm{~mm}$ for example) and a given conductivity $\left(\sigma=5.9 \times 10^{7} \frac{\mathrm{A}}{\mathrm{Vm}}\right.$ for copper $)$. However, this asymptotic point of view is known for similar problems to be rather efficient $[3,12]$ to design numerical methods. Moreover, scaling $\omega \mu_{0} \sigma$ like $1 / \varepsilon$ corresponds to a borderline case where the sheet is neither impenetrable (this will happen for $\left|\omega \mu_{0} \sigma\right|^{-1}=o(\varepsilon)$ ) nor transparent $\left(\left|\omega \mu_{0} \sigma\right|=o\left(\varepsilon^{-1}\right)\right)$ in the limit for $\varepsilon$ going to 0 . With the scaling $\omega \mu_{0} \sigma=\frac{c_{0}}{\varepsilon}$ already the limit model for $\varepsilon \rightarrow 0$ is physically relevant and an asymptotic expansion is expected to be accurate already with a few terms.

\subsection{Objective of the paper}

In what follows we will design a one step procedure to compute a numerical approximation of $e^{\varepsilon}$ which does require neither mesh refinement nor meshing of the thin sheet $\Omega_{\mathrm{int}}^{\varepsilon}$. This technique is based on the asymptotic expansion for $\varepsilon$ varying to 0 of $e^{\varepsilon}$ obtained in [24]. It consists in modelling the thin sheet by two approximate transmission conditions which are derived and justified in three steps:

(i) We derive formally an approximate models whose solutions $\widetilde{e}^{\varepsilon, N}$ are candidates to approximate the exact solution $e^{\varepsilon}$.

(ii) We prove that the approximate problems are well posed for small $\varepsilon$ and asymptotically stable.

(iii) We prove that $\widetilde{e}^{\varepsilon, N}$ is an approximation of $e^{\varepsilon}$ of order $N$, i.e., $\widetilde{e}^{\varepsilon, N}-e^{\varepsilon}=\underset{\varepsilon \rightarrow 0}{o}\left(\varepsilon^{N}\right)$.

In order to have a presentation as clear as possible, this article will only carry on the cases corresponding to approximation order $N \leq 3$. These results can be extended to $N>3$ even if one has to deal with higher derivative operators on the midline $\Gamma$ of the sheet which introduces extra difficulties. The role of steps (ii) and (iii) is to give a mathematical background to the formal computations of step (i). In Section 2 the result of [24] will be shortly summarised. The steps (i) will be formally carried out in Section 3 for any order $N$. Section 4 is devoted to the concrete transmission condition for order $N=1$ including steps (ii) and (iii), while Section 5 deals with order $N=2$ and $N=3$. Finally, in Section 6 numerical experiments will be shown.

\section{HiERARCHICAL ASYMPTOTIC EXPANSIONS}

We aim in this section in summarising the results of [24], where we have derived the complete asymptotic expansion with respect to the width of the sheet $\varepsilon$ of the solution of problem (1.12) for a regular sheet. 
This complete asymptotic expansion consists of two parts. First, the restriction of $e^{\varepsilon}$ to the exterior of the sheet has been considered where we were seeking for a Taylor expansion of this restriction:

$$
e_{\text {ext }}^{\varepsilon}(\underline{x})=\left.e^{\varepsilon}\right|_{\Omega_{\mathrm{ext}}^{\varepsilon}}(\underline{x})=e_{\mathrm{ext}}^{\varepsilon, N}(\underline{x})+r_{\mathrm{ext}}^{\varepsilon, N}(\underline{x}) \quad \text { with } \quad e_{\mathrm{ext}}^{\varepsilon, N}(\underline{x}):=\sum_{j=0}^{N} \varepsilon^{j} u_{\mathrm{ext}}^{j}(\underline{x}), \quad \forall N \in \mathbb{N} .
$$

Secondly, we considered the restriction to the interior of the sheet of $e^{\varepsilon}$. For this restriction we were not searching for a Taylor expansion of $e^{\varepsilon}$ in the original curvilinear coordinates $(s, t)$, but in the normalised curvilinear coordinates $(S, t)=\left(\frac{s}{\varepsilon}, t\right)$

$$
e_{\mathrm{int}}^{\varepsilon}(\underline{x})=\left.e^{\varepsilon}\right|_{\Omega_{\mathrm{int}}^{\varepsilon}}(\underline{x})=e_{\mathrm{int}}^{\varepsilon, N}(\underline{x})+r_{\mathrm{int}}^{\varepsilon, N}(\underline{x}) \text { with } e_{\mathrm{int}}^{\varepsilon, N}(s, t):=\sum_{j=0}^{N} \varepsilon^{j} u_{\mathrm{int}}^{j}\left(\frac{s}{\varepsilon}, t\right), \quad \forall N \in \mathbb{N} .
$$

The following two estimates make clear what we mean by Taylor expansion

$$
\begin{aligned}
& \left\|e_{\mathrm{ext}}^{\varepsilon}-e_{\mathrm{ext}}^{\varepsilon, N}\right\|_{H^{1}\left(\Omega_{\mathrm{ext}}^{\varepsilon}\right)}=\left\|r_{\mathrm{ext}}^{\varepsilon, N}\right\|_{H^{1}\left(\Omega_{\mathrm{ext}}^{\varepsilon}\right)} \leq C_{N} \varepsilon^{N+1}, \quad \forall N \in \mathbb{N}, \\
& \left\|e_{\mathrm{int}}^{\varepsilon}-e_{\mathrm{int}}^{\varepsilon, N}\right\|_{H^{1}\left(\Omega_{\mathrm{int}}^{\varepsilon}\right)}=\left\|r_{\mathrm{int}}^{\varepsilon, N}\right\|_{H^{1}\left(\Omega_{\mathrm{int}}^{\varepsilon}\right)} \leq C_{N} \varepsilon^{N+\frac{1}{2}}, \quad \forall N \in \mathbb{N} .
\end{aligned}
$$

The coefficients $u_{\text {ext }}^{j}(\underline{x})$ and $u_{\text {int }}^{j}(S, t)$ of the above Taylor expansions are functions, which do not depend on $\varepsilon$ and which are defined on the limit domain $\Omega \backslash \Gamma$ of $\Omega_{\text {ext }}^{\varepsilon}$ for $\varepsilon \rightarrow 0$ and on the normalised sub-domain of the sheet $\hat{\Gamma} \times\left[-\frac{1}{2}, \frac{1}{2}\right]$, respectively. In [24], the terms of the exterior expansion and interior expansions $u_{\text {ext }}^{j}$ and $u_{\text {int }}^{j}$ have been defined order by order by a coupled problem. The $u_{\text {int }}^{j}$ were then eliminated - this has been done explicitly up to order 2 in [24] - to obtain a definition of $u_{\text {ext }}^{j}$ with problems involving only the exterior coefficients of lower order and not the interior coefficients. These hierarchical decoupled problems take the form

$$
\begin{aligned}
& u_{\mathrm{ext}}^{j} \in H_{\Delta}^{1}(\Omega \backslash \Gamma):=\left\{u \in H^{1}(\Omega \backslash \Gamma): \Delta u \in L^{2}(\Omega \backslash \Gamma) \text { and } u=0 \text { on } \partial \Omega\right\}, \\
& -\Delta u_{\mathrm{ext}}^{j}(\underline{x})=f_{j}(\underline{x}), \quad \text { in } \Omega \backslash \Gamma, \\
& {\left[u_{\mathrm{ext}}^{j}\right](t)=\sum_{\ell=2}^{j}\left(\gamma_{\ell} u_{\mathrm{ext}}^{j-\ell}\right)(t), \quad \text { on } \Gamma,} \\
& {\left[\partial_{n} u_{\text {ext }}^{j}\right](t)-\mathrm{i} c_{0}\left\{u_{\text {ext }}^{j}\right\}(t)=\sum_{\ell=1}^{j}\left(\zeta_{\ell} u_{\text {ext }}^{j-\ell}\right)(t), \quad \text { on } \Gamma,}
\end{aligned}
$$

with

- the two transmission operators $[\cdot]$ and $\{\cdot\}$ defined on the midline $\Gamma$ by

$$
[u](t):=u\left(t, 0^{+}\right)-u\left(t, 0^{-}\right), \quad\{u\}(t):=\frac{1}{2}\left(u\left(t, 0^{+}\right)+u\left(t, 0^{-}\right)\right)
$$

- the differential operators $\gamma_{\ell}$ and $\boldsymbol{\zeta}_{\ell}$ that are explicitly given for $\ell \leq 3$ in Appendix A.1;

- the source terms $f_{j}$ that are inherited from the original problem and consequently satisfy $(j>0)$

$$
f_{0}(\underline{x})=f(\underline{x}) \quad \text { and } \quad f_{j}(\underline{x})=0, \quad \text { in } \Omega \backslash \Gamma
$$

- the spaces $H^{m}(\Omega \backslash \Gamma)$, for all $m \in \mathbb{N}$, which are the sets of all functions $u$ which are $H^{m}\left(\Omega_{+}\right)$and $H^{m}\left(\Omega_{-}\right)$where $\Omega_{+}$and $\Omega_{-}$are the two connected component of $\Omega \backslash \Gamma$ (see Fig. 1 ). 
Remark 2.1. For $j=0$ and 1 , the functions $u_{\text {ext }}^{j}$ are continuous across the interface $\Gamma$ but not $C^{1}(\Omega)$. For $j \geq 2$, the functions $u_{\mathrm{ext}}^{j}$ are not continuous across the interface $\Gamma$. However, they are well-defined and regular on each connected component $\Omega_{+}$and $\Omega_{-}$of $\Omega \backslash \Gamma$, see Appendix B.

Once the exterior coefficients are defined the interior coefficients can be computed as combinations of the expansion terms for the exterior fields at the same and previous orders

$$
u_{\text {int }}^{j}(t, S)=\sum_{\ell=0}^{j}\left(\boldsymbol{\eta}_{\ell} u_{\mathrm{ext}}^{j-\ell}\right)(t, S),
$$

where the differential operators $\boldsymbol{\eta}_{\ell}$ are given for $\ell \leq 3$ in Appendix A.2. The interior coefficients are polynomials in the normal coordinate $S$ and result by the exterior fields of the same and the previous orders.

Remark 2.2. The latter asymptotic expansion can directly be used to obtain a numerical approximation of $e^{\varepsilon}$. Indeed one has just to compute $e_{\mathrm{ext}}^{\varepsilon, N}$ and $e_{\mathrm{int}}^{\varepsilon, N}$, with $N$ fixed by the desired precision. These computations do require neither mesh refinement nor the meshing of the thin sheet. However, this method suffers from a major drawback: for relatively large $\varepsilon$ the model of order 0 does quite possibly not reach the desired precision and one has to compute further terms of the asymptotic expansion in order to obtain a sharp approximation to $e^{\varepsilon}$. The multi-step procedure is not standard and may disencourage to be implemented in a numerical library.

\section{DERIVATION OF THE TRANSMISSION CONDITIONS FOR THE EXTERIOR FIELDS}

This section is devoted to the derivation of transmission conditions for general orders based on the terms $u_{\mathrm{ext}}^{j}$ of the asymptotic expansion of the exterior part of the exact solution $e^{\varepsilon}$. These transmission conditions with associated problems define approximate functions $\tilde{e}^{\varepsilon, N}$ of a particular order $N$ in one step.

\subsection{The definition of the exterior approximation}

We adopt the point of view of formal series. Due to (2.1), the formal Taylor series of $e_{\text {ext }}^{\varepsilon}$ takes the form

$$
e_{\mathrm{ext}}^{\varepsilon}(\underline{x}) \sim \sum_{j=0}^{+\infty} \varepsilon^{j} u_{\mathrm{ext}}^{j}(\underline{x})
$$

where we have adopted the symbol " " to mention that this series may diverge or converge but not toward $e_{\mathrm{ext}}^{\varepsilon}$. Therefore multiplying for all $j \in \mathbb{N}$ system $(2.5 \mathrm{c}),(2.5 \mathrm{~d})$ by $\varepsilon^{j}$ and summing we get with

$$
\begin{aligned}
{\left[\sum_{j=0}^{+\infty} \varepsilon^{j} u_{\mathrm{ext}}^{j}\right](t) } & =\sum_{j=0}^{+\infty} \varepsilon^{j} \sum_{\ell=0}^{j}\left(\gamma_{\ell} u_{\mathrm{ext}}^{j-\ell}\right)(t), \quad \text { on } \Gamma, \\
{\left[\partial_{n} \sum_{j=0}^{+\infty} \varepsilon^{j} u_{\mathrm{ext}}^{j}\right](t)-\mathrm{i} c_{0}\left\{\sum_{j=0}^{+\infty} \varepsilon^{j} u_{\mathrm{ext}}^{j}\right\}(t) } & =\sum_{j=0}^{+\infty} \varepsilon^{j} \sum_{\ell=0}^{j}\left(\boldsymbol{\zeta}_{\ell} u_{\mathrm{ext}}^{j-\ell}\right)(t), \quad \text { on } \Gamma,
\end{aligned}
$$

with the convention $\gamma_{0}=\gamma_{1}=\zeta_{0}=0$. Interchanging the two sums and identifying $e_{\mathrm{ext}}^{\varepsilon}$, we find

$$
\begin{aligned}
{\left[e_{\mathrm{ext}}^{\varepsilon}\right](t) } & \sim\left(\boldsymbol{\gamma}^{\varepsilon} e_{\mathrm{ext}}^{\varepsilon}\right)(t), & & \text { on } \Gamma, \\
{\left[\partial_{n} e_{\mathrm{ext}}^{\varepsilon}\right](t)-\mathrm{i} c_{0}\left\{e_{\mathrm{ext}}^{\varepsilon}\right\}(t) } & \sim\left(\boldsymbol{\zeta}^{\varepsilon} e_{\mathrm{ext}}^{\varepsilon}\right)(t), & & \text { on } \Gamma,
\end{aligned}
$$

with the two formal operator series $\gamma^{\varepsilon}$ and $\boldsymbol{\zeta}^{\varepsilon}$ given by

$$
\boldsymbol{\gamma}^{\varepsilon}=\sum_{j=0}^{+\infty} \varepsilon^{j} \gamma_{j}, \quad \text { and } \quad \boldsymbol{\zeta}^{\varepsilon}=\sum_{j=0}^{+\infty} \varepsilon^{j} \boldsymbol{\zeta}_{j}
$$


These two transmission conditions appear to be perfect. However, the question of convergence of the series (3.4) remains (they diverge potentially). Moreover, it seems not possible to get a simple formula for the sum if it exists. Consequently, these two perfect transmission conditions could not directly be used for numerical computations. However, truncating these two series at a given order $N$

$$
\boldsymbol{\gamma}^{\varepsilon, N}=\sum_{j=0}^{N} \varepsilon^{j} \boldsymbol{\gamma}_{j}, \text { and } \boldsymbol{\zeta}^{\varepsilon, N}=\sum_{j=0}^{N} \varepsilon^{j} \boldsymbol{\zeta}_{j}
$$

we get well defined transmission conditions to model the highly conductive thin sheet.

This series truncation leads to problems for the approximate solutions $\tilde{e}_{\text {ext }}^{\varepsilon, N}$

$$
\begin{aligned}
\tilde{e}_{\mathrm{ext}}^{\varepsilon, N} & \in H_{\Delta}^{1}(\Omega \backslash \Gamma), & & \\
-\Delta \tilde{e}_{\mathrm{ext}}^{\varepsilon, N}(\underline{x}) & =f(\underline{x}), & & \text { in } \Omega \backslash \Gamma, \\
{\left[\tilde{e}_{\mathrm{ext}}^{\varepsilon, N}\right](t)-\left(\boldsymbol{\gamma}^{\varepsilon, N} \tilde{e}_{\mathrm{ext}}^{\varepsilon, N}\right)(t) } & =0, & & \text { on } \Gamma, \\
{\left[\partial_{n} \tilde{e}_{\mathrm{ext}}^{\varepsilon, N}\right](t)-\mathrm{i} c_{0}\left\{\tilde{e}_{\mathrm{ext}}^{\varepsilon, N}\right\}(t)-\left(\boldsymbol{\zeta}^{\varepsilon, N} \tilde{e}_{\mathrm{ext}}^{\varepsilon, N}(t)\right) } & =0, & & \text { on } \Gamma .
\end{aligned}
$$

The approximate solutions are indexed by $N$ which is related to the order of the approximation. The reader may note that the approximate solution for $N>1$ is no more continuous across $\Gamma$ and therefore does not belong to $H^{1}(\Omega)$, but to $H^{1}(\Omega \backslash \Gamma)$.

The question of existence and uniqueness of $\widetilde{e}^{\varepsilon, N}$ is not only an interesting mathematical question. Very often problems arising from an asymptotic expansion are not well posed and so not well suited for numerical computations. Consequently, it is crucial to check the existence and uniqueness of solution of problem (3.6). Moreover, the convergence proof needs a stability result that is closely related to the existence and uniqueness of the solution of the collected models. We will come to this question in Section 4 for order $N=1$ and in Section 5 for order $N=2$.

\subsection{The definition of the interior approximation}

The same strategy can be applied to the derivation of an interior approximation. Due to (2.2), the Taylor series of $e_{\text {int }}^{\varepsilon}$ reads $e_{\text {int }}^{\varepsilon}(t, s) \sim \sum_{j=0}^{+\infty} \varepsilon^{j} u_{\text {int }}^{j}\left(t, \frac{s}{\varepsilon}\right)$. Inserting (2.7) we have

$$
e_{\mathrm{int}}^{\varepsilon}(t, s) \sim \sum_{j=0}^{+\infty} \varepsilon^{j} \sum_{\ell=0}^{j}\left(\boldsymbol{\eta}_{\ell} u_{\mathrm{ext}}^{j-\ell}\right)\left(t, \frac{s}{\varepsilon}\right)=\left(\sum_{\ell=0}^{+\infty} \varepsilon^{\ell} \boldsymbol{\eta}_{\ell}\right)\left(\sum_{j=0}^{+\infty} \varepsilon^{j} u_{\mathrm{ext}}^{j}\right)\left(t, \frac{s}{\varepsilon}\right) \sim \boldsymbol{\eta}^{\varepsilon} e_{\mathrm{ext}}^{\varepsilon}\left(t, \frac{s}{\varepsilon}\right)
$$

with the operator series $\boldsymbol{\eta}^{\varepsilon}$, which is the formal limit for $N \rightarrow+\infty$ of

$$
\boldsymbol{\eta}^{\varepsilon, N}:=\sum_{\ell=0}^{N} \varepsilon^{\ell} \boldsymbol{\eta}_{\ell}
$$

Using this operator for a concrete $N$ leads to the introduction of the interior approximation of order $N$

$$
\tilde{e}_{\text {int }}^{\varepsilon, N}(t, s)=\left(\boldsymbol{\eta}^{\varepsilon, N} \tilde{e}_{\text {ext }}^{\varepsilon, N}\right)\left(t, \frac{s}{\varepsilon}\right) .
$$

Remark 3.1. In the continuation we will not carry on the justification of these approximations. Note, however, that it can be proved that $\tilde{e}_{\text {int }}^{\varepsilon, N}$ is an approximation of order $N-\frac{1}{2}$ of $e_{\mathrm{int}}^{\varepsilon}$, i.e., (see also Figs. 5 and 6)

$$
\left\|e_{\mathrm{int}}^{\varepsilon}-\tilde{e}_{\mathrm{int}}^{\varepsilon, N}\right\|_{H^{1}\left(\Omega_{\mathrm{int}}^{\varepsilon}\right)}=\left\|r_{\mathrm{int}}^{\varepsilon, N}\right\|_{H^{1}\left(\Omega_{\mathrm{int}}^{\varepsilon}\right)} \leq C_{N} \varepsilon^{N+\frac{1}{2}} .
$$




\section{The TRANSMISSION CONDITIONS OF ORDER 1}

The system (3.6) with the transmission conditions of order 1 is given by

$$
\begin{array}{rlrl}
\tilde{e}_{\mathrm{ext}}^{\varepsilon, 1} & \in H_{\Delta}^{1}(\Omega), & \\
-\Delta \tilde{e}_{\mathrm{ext}}^{\varepsilon, 1}(\underline{x}) & =f(\underline{x}), & & \text { in } \Omega \backslash \Gamma, \\
{\left[\tilde{e}_{\mathrm{ext}}^{\varepsilon, 1}\right](t)} & =0, & & \text { on } \Gamma . \\
{\left[\partial_{n} \tilde{e}_{\mathrm{ext}}^{\varepsilon, 1}\right](t)-\left(\mathrm{i} c_{0}-\varepsilon \frac{c_{0}^{2}}{6}\right)\left\{\tilde{e}_{\mathrm{ext}}^{\varepsilon, 1}\right\}(t)} & =0, & & \text { on } \Gamma .
\end{array}
$$

With the vanishing jump (4.1c) we may use $\tilde{e}_{\text {ext }}^{\varepsilon, 1}$ equivalently to $\left\{\tilde{e}_{\text {ext }}^{\varepsilon, 1}\right\}$ for convenience.

\subsection{The weak formulation}

In what follows, homogeneous Dirichlet boundary conditions at $\partial \Omega$ will be incorporated in the trial and test spaces which are

$$
H_{\partial \Omega}^{1}(\Omega)=\left\{v \in H^{1}(\Omega): v=0 \text { on } \partial \Omega\right\}
$$

Hence, the system (4.1) is equivalent to the variational formulation: Seek $\tilde{e}_{\text {ext }}^{\varepsilon, 1} \in H_{\partial \Omega}^{1}(\Omega)$ such that

$$
\mathrm{a}_{1}^{\varepsilon}\left(\tilde{e}_{\mathrm{ext}}^{\varepsilon, 1}, e^{\prime}\right)=\left\langle\ell, e^{\prime}\right\rangle, \text { for all } e^{\prime} \in H_{\partial \Omega}^{1}(\Omega)
$$

with the bilinear form $a_{1}^{\varepsilon}$ and the linear form $\ell$ defined by

$$
\begin{aligned}
& \mathrm{a}_{1}^{\varepsilon}\left(e, e^{\prime}\right)=\int_{\Omega} \nabla e \cdot \nabla \overline{e^{\prime}} \mathrm{d} \underline{x}+\int_{\Gamma}\left(\mathrm{i} c_{0}-\varepsilon \frac{c_{0}^{2}}{6}\right)\{e\}\left\{\overline{e^{\prime}}\right\} \mathrm{d} t, \\
& \left\langle\ell, e^{\prime}\right\rangle=\int_{\Omega} f \overline{e^{\prime}} \mathrm{d} \underline{x} .
\end{aligned}
$$

\subsection{Well-posedness and stability}

Lemma 4.1. There exists a constant $\gamma>0$ such that for all $\varepsilon>0$ and for all $e \in H_{\partial \Omega}^{1}(\Omega)$ we have

$$
\left|\mathrm{a}_{1}^{\varepsilon}(e, e)\right| \geq \frac{\gamma}{\sqrt{1+c_{0}^{2} \varepsilon^{2}}}\|e\|_{H^{1}(\Omega)}^{2} .
$$

Proof. Since $\left|1-\mathrm{i} c_{0} \varepsilon\right|=\sqrt{1+c_{0}^{2} \varepsilon^{2}}$ and by inserting $\mathrm{a}_{1}^{\varepsilon}(e, e)$, we have

$$
\left|\mathrm{a}_{1}^{\varepsilon}(e, e)\right|=\left|\frac{1-\mathrm{i} c_{0} \varepsilon}{\sqrt{1+c_{0}^{2} \varepsilon^{2}}} \mathrm{a}_{1}^{\varepsilon}(e, e)\right| \geq \operatorname{Re}\left(\frac{1-\mathrm{i} c_{0} \varepsilon}{\sqrt{1+c_{0}^{2} \varepsilon^{2}}} \mathrm{a}_{1}^{\varepsilon}(e, e)\right) \geq \frac{1}{\sqrt{1+c_{0}^{2} \varepsilon^{2}}}\left(|e|_{H^{1}(\Omega)}^{2}+\frac{5}{6} c_{0}^{2} \varepsilon\|e\|_{L^{2}(\Gamma)}^{2}\right)
$$

By positivity of $\|e\|_{L^{2}(\Gamma)}^{2}$ and Poincaré-Friedrichs inequality [6] since $e \in H_{\partial \Omega}^{1}(\Omega)$, it follows for some $\gamma>0$

$$
\left|a_{1}^{\varepsilon}(e, e)\right| \geq \frac{1}{\sqrt{1+c_{0}^{2} \varepsilon^{2}}}|e|_{H^{1}(\Omega)}^{2} \geq \frac{\gamma}{\sqrt{1+c_{0}^{2} \varepsilon^{2}}}\|e\|_{H^{1}(\Omega)}^{2} .
$$

The proof is complete. 
Remark 4.2. This proof can easily be adapted to other boundary conditions. For the Neumann boundary condition, where we search for solutions $e \in H^{1}(\Omega)$, we can use the estimate

$$
\left|\mathrm{a}_{1}^{\varepsilon}(e, e)\right| \geq \frac{\left|\operatorname{Re}\left(\frac{1-\mathrm{i} c_{0} \varepsilon}{\sqrt{1+c_{0}^{2} \varepsilon^{2}}} \mathrm{a}_{1}^{\varepsilon}(e, e)\right)\right|+\left|\operatorname{Im}\left(\mathrm{a}_{1}^{\varepsilon}(e, e)\right)\right|}{2} .
$$

Then, the steps for the real part in last proof are repeated, and after noting that $\operatorname{Im}\left(\mathrm{a}_{1}^{\varepsilon}(e, e)\right)=c_{0}\|e\|_{L^{2}(\Gamma)}^{2}$. Finally, the following Poincaré-Friedrichs inequality in $H^{1}(\Omega)$ can be applied

$$
|e|_{H^{1}(\Omega)}^{2}+c_{0}\|e\|_{L^{2}(\Gamma)}^{2} \geq \gamma^{\prime}\|e\|_{H^{1}(\Omega)}^{2}, \quad \forall e \in H^{1}(\Omega)
$$

A direct consequence of the coercivity and of the Lax-Milgram lemma [6] is the existence and uniqueness of the solution of the model of order 1 .

Lemma 4.3. There exists a unique solution $\tilde{e}_{\mathrm{ext}}^{\varepsilon, 1} \in H_{\partial \Omega}^{1}(\Omega)$ of (4.3) which satisfies

$$
\left\|\tilde{e}_{\text {ext }}^{\varepsilon, 1}\right\|_{H^{1}(\Omega)} \leq \frac{\sqrt{1+c_{0}^{2} \varepsilon^{2}}}{\gamma}\|\ell\|_{\left(H_{\partial \Omega}^{1}(\Omega)\right)^{\prime}} .
$$

\subsection{Estimates of the modelling error}

In this section we show that the solution of the model of order 1 approximates the exact solution with an error of order $\varepsilon^{2}$.

Theorem 4.4 (modelling error for order 1). There exists a constant $C$ independent of $\varepsilon$ such that

$$
\left\|\tilde{e}_{\mathrm{ext}}^{\varepsilon, 1}-e_{\mathrm{ext}}^{\varepsilon}\right\|_{H^{1}\left(\Omega_{\mathrm{ext}}^{\varepsilon}\right)}=\underset{\varepsilon \rightarrow 0}{O}\left(\varepsilon^{2}\right)
$$

Proof. Both functions $\tilde{e}_{\mathrm{ext}}^{\varepsilon, 1}$ and $e_{\mathrm{ext}}^{\varepsilon}$ will be compared to the exterior asymptotic expansion truncated at order 1

$$
e_{\mathrm{ext}}^{\varepsilon, 1}=u_{\mathrm{ext}}^{0}+\varepsilon u_{\mathrm{ext}}^{1} .
$$

Due to the triangular inequality, we have

$$
\left\|\tilde{e}_{\mathrm{ext}}^{\varepsilon, 1}-e_{\mathrm{ext}}^{\varepsilon}\right\|_{H^{1}\left(\Omega_{\mathrm{ext}}^{\varepsilon}\right)} \leq\left\|\tilde{e}_{\mathrm{ext}}^{\varepsilon, 1}-e_{\mathrm{ext}}^{\varepsilon, 1}\right\|_{H^{1}\left(\Omega_{\mathrm{ext}}^{\varepsilon}\right)}+\left\|e_{\mathrm{ext}}^{\varepsilon, 1}-e_{\mathrm{ext}}^{\varepsilon}\right\|_{H^{1}\left(\Omega_{\mathrm{ext}}^{\varepsilon}\right)} .
$$

We shall show that both terms are $\underset{\varepsilon \rightarrow 0}{O}\left(\varepsilon^{2}\right)$.

(i) To estimate the first term, we remark that $e_{\text {ext }}^{\varepsilon, 1}$ solves

$$
\begin{array}{rlrl}
e_{\mathrm{ext}}^{\varepsilon, 1} & \in H_{\Delta}^{1}(\Omega), & \\
-\Delta e_{\mathrm{ext}}^{\varepsilon, 1}(\underline{x}) & =f(\underline{x}), & & \text { in } \Omega \backslash \Gamma, \\
{\left[e_{\mathrm{ext}}^{\varepsilon, 1}\right](t)} & =0, & & \text { on } \Gamma . \\
{\left[\partial_{n} e_{\mathrm{ext}}^{\varepsilon, 1}\right](t)-\left(\mathrm{i} c_{0}-\varepsilon \frac{c_{0}^{2}}{6}\right)\left\{e_{\mathrm{ext}}^{\varepsilon, 1}\right\}(t)} & =\varepsilon^{2} \frac{c_{0}^{2}}{6} u_{\mathrm{ext}}^{1}(t), & & \text { on } \Gamma .
\end{array}
$$

Comparing (4.15) to (4.1), we observe that the error function $\tilde{e}_{\text {ext }}^{\varepsilon, 1}-e_{\text {ext }}^{\varepsilon, 1} \in H_{\partial \Omega}^{1}(\Omega)$ is solution of the variational formulation

$$
\mathrm{a}_{1}^{\varepsilon}\left(\tilde{e}_{\mathrm{ext}}^{\varepsilon, 1}-e_{\mathrm{ext}}^{\varepsilon, 1}, e^{\prime}\right)=\ell_{1}^{\varepsilon}\left(e^{\prime}\right) \quad \forall e^{\prime} \in H_{\partial \Omega}^{1}(\Omega),
$$


with

$$
\ell_{1}^{\varepsilon}\left(e^{\prime}\right):=-\varepsilon^{2} \frac{c_{0}^{2}}{6} \int_{\Gamma} u_{\mathrm{ext}}^{1}(t) e^{\prime}(t) \mathrm{d} t
$$

Due to the Cauchy-Schwarz inequality and the trace theorem we have for all $e^{\prime} \in H_{\partial \Omega}^{1}(\Omega) \subset H^{1}(\Omega)$

$$
\ell_{1}^{\varepsilon}\left(e^{\prime}\right) \leq \varepsilon^{2} \frac{c_{0}^{2}}{6}\left\|u_{\mathrm{ext}}^{1}\right\|_{L^{2}(\Gamma)}\left\|e^{\prime}(t)\right\|_{L^{2}(\Gamma)} \leq C \varepsilon^{2}\left\|u_{\mathrm{ext}}^{1}\right\|_{L^{2}(\Gamma)}\left\|e^{\prime}\right\|_{H^{1}(\Omega)} .
$$

Since the function $u_{\mathrm{ext}}^{1}$ does not depend on $\varepsilon$, we obtain

$$
\left\|\ell_{1}^{\varepsilon}\right\|_{\left(H_{\partial \Omega}^{1}(\Omega)\right)^{\prime}}=\sup _{e^{\prime} \in H_{\partial \Omega}^{1}(\Omega)} \frac{\ell_{1}^{\varepsilon}\left(e^{\prime}\right)}{\left\|e^{\prime}\right\|_{H^{1}(\Omega)}}=\operatorname{O}_{\varepsilon \rightarrow 0}\left(\varepsilon^{2}\right)
$$

and with the stability estimate (4.11) of the variational formulation we conclude

$$
\left\|\tilde{e}_{\mathrm{ext}}^{\varepsilon, 1}-e_{\mathrm{ext}}^{\varepsilon, 1}\right\|_{H^{1}\left(\Omega_{\mathrm{ext}}^{\varepsilon}\right)}=\underset{\varepsilon \rightarrow 0}{O}\left(\varepsilon^{2}\right) .
$$

(ii) The estimate for the second term follows from (2.3).

\section{The TRANSMission CONDITIONS OF ORDER 2 AND ORDER 3}

In this section, a weak formulation for the models of order 2 and 3 will be derived. Their solutions solve transmission problems (3.6) written for $N=2$ and 3 that involve the operators $\gamma_{j}$ and $\boldsymbol{\zeta}_{j}$ for $j \leq 3$, which are given in (A.1) and (A.2). More explicitly, the transmission conditions are given for $N=2$ by

$$
\begin{aligned}
& {\left[\tilde{e}_{\text {ext }}^{\varepsilon, 2}\right](t)+\varepsilon^{2} \frac{\mathrm{i} c_{0} \kappa(t)}{24}\left\{\tilde{e}_{\text {ext }}^{\varepsilon, 2}\right\}(t)+\varepsilon^{2} \frac{\mathrm{i} c_{0}}{12}\left\{\partial_{n} \tilde{e}_{\text {ext }}^{\varepsilon, 2}\right\}(t)=0,} \\
& {\left[\partial_{n} \tilde{e}_{\text {ext }}^{\varepsilon, 2}\right](t)-\left(\mathrm{i} c_{0}-\varepsilon \frac{c_{0}^{2}}{6}-\varepsilon^{2} \frac{\mathrm{i} c_{0}}{12}\left(\frac{7 c_{0}^{2}}{20}+\partial_{t}^{2}\right)\right)\left\{\tilde{e}_{\text {ext }}^{\varepsilon, 2}\right\}(t)-\varepsilon^{2} \frac{\mathrm{i} c_{0} \kappa(t)}{24}\left\{\partial_{n} \tilde{e}_{\text {ext }}^{\varepsilon, 2}\right\}(t)=0,}
\end{aligned}
$$

and for $N=3$ by

$$
\begin{array}{r}
{\left[\tilde{e}_{\text {ext }}^{\varepsilon, 3}\right](t)+\varepsilon^{2} \frac{\mathrm{i} c_{0} \kappa(t)}{24}\left(1-\varepsilon \frac{\mathrm{i} c_{0}}{10}\right)\left\{\tilde{e}_{\mathrm{ext}}^{\varepsilon, 3}\right\}(t)+\varepsilon^{2} \frac{\mathrm{i} c_{0}}{12}\left(1-\varepsilon \frac{\mathrm{i} c_{0}}{10}\right)\left\{\partial_{n} \tilde{e}_{\mathrm{ext}}^{\varepsilon, 3}\right\}(t)=0} \\
{\left[\partial_{n} \tilde{e}_{\mathrm{ext}}^{\varepsilon, 3}\right](t)-\left(\mathrm{i} c_{0}-\varepsilon \frac{c_{0}^{2}}{6}-\varepsilon^{2} \frac{\mathrm{i} c_{0}}{12}\left(\frac{7 c_{0}^{2}}{20}+\partial_{t}^{2}\right)\right.} \\
\left.+\varepsilon^{3} \frac{c_{0}^{2}}{40}\left(\frac{17 c_{0}^{2}}{84}+\frac{\kappa^{2}(t)}{3}+\partial_{t}^{2}\right)\right)\left\{\tilde{e}_{\mathrm{ext}}^{\varepsilon, 3}\right\}(t) \\
-\varepsilon^{2} \frac{\mathrm{i} c_{0} \kappa(t)}{24}\left(1-\varepsilon \frac{\mathrm{i} c_{0}}{10}\right)\left\{\partial_{n} \tilde{e}_{\mathrm{ext}}^{\varepsilon, 3}\right\}(t)=0 .
\end{array}
$$

The transmission conditions of order 2 and 3 are rather similar. They differ only by terms of order $\varepsilon^{3}$. In the continuation, we will give most of the details for the model of order 2 and will only state the result for the model of order 3 to avoid repetitions.

\subsection{The weak formulation: a mixed formulation}

We first derive the variational formulation for the model of order 2. With the first transmission condition (5.1a), the solution of the approximate models of order 2 is in general not continuous across the interface $\Gamma$. The natural functional framework is not anymore $H_{\partial \Omega}^{1}(\Omega)$ but

$$
H_{\partial \Omega}^{1}(\Omega \backslash \Gamma)=\left\{v \in H^{1}(\Omega \backslash \Gamma): v=0 \text { on } \partial \Omega\right\} .
$$


The derivation of the variational formulation is based on the following Green formula

$$
\int_{\Omega \backslash \Gamma} \Delta e \overline{e^{\prime}} \mathrm{d} \underline{x}+\int_{\Omega \backslash \Gamma} \nabla e \cdot \nabla \overline{e^{\prime}} \mathrm{d} \underline{x}+\int_{\Gamma}\left[\partial_{n} e\right]\left\{\overline{e^{\prime}}\right\}+\left\{\partial_{n} e\right\}\left[\overline{e^{\prime}}\right] \mathrm{d} t=0, \quad \forall e \in C^{\infty}(\Omega \backslash \Gamma) \text { and } e^{\prime} \in H_{\partial \Omega}^{1}(\Omega \backslash \Gamma) .
$$

For $e=\widetilde{e}_{\text {ext }}^{\varepsilon, 2}$, the two transmissions conditions (5.1) can easily be inserted in (5.4) to eliminate $\partial_{n} e$. However, this leads to an $\varepsilon$-degenerated variational formulation with $\varepsilon^{-2}$ factors. Therefore, we prefer to replace the mean normal trace in (5.4) as well as in (5.1) by a new unknown function

$$
\tilde{\lambda}_{\text {ext }}^{\varepsilon, 2}=\left\{\partial_{n} \tilde{e}_{\text {ext }}^{\varepsilon, 2}\right\} \in L^{2}(\Gamma) .
$$

We shall now derive a system governing $e=\tilde{e}_{\text {ext }}^{\varepsilon, 2}$ and $\tilde{\lambda}_{\text {ext }}^{\varepsilon, 2}$. Inserting (5.5) and (5.1b) in (5.4) leads to

$$
\begin{aligned}
\int_{\Omega \backslash \Gamma} \nabla \tilde{e}_{\mathrm{ext}}^{\varepsilon, 2} \cdot \nabla \overline{e^{\prime}} \mathrm{d} \underline{x}+\left(\mathrm{i} c_{0}-\varepsilon \frac{c_{0}^{2}}{6}-\varepsilon^{2} \frac{7 \mathrm{i} c_{0}^{3}}{240}\right)\left\langle\left\{\tilde{e}_{\mathrm{ext}}^{\varepsilon, 2}\right\},\left\{e^{\prime}\right\}\right\rangle_{\Gamma}-\varepsilon^{2} \frac{\mathrm{i} c_{0}}{12}\left\langle\partial_{t}^{2}\left\{\tilde{e}_{\mathrm{ext}}^{\varepsilon, 2}\right\},\left\{e^{\prime}\right\}\right\rangle_{\Gamma} & \\
& +\varepsilon^{2} \frac{\mathrm{i} c_{0}}{24}\left\langle\kappa \tilde{\lambda}_{\mathrm{ext}}^{\varepsilon, 2},\left\{e^{\prime}\right\}\right\rangle_{\Gamma}+\left\langle\tilde{\lambda}_{\mathrm{ext}}^{\varepsilon, 2},\left[e^{\prime}\right]\right\rangle_{\Gamma}=\left\langle f, e^{\prime}\right\rangle,
\end{aligned}
$$

where $\langle\cdot, \cdot\rangle_{\Gamma}$ denotes the inner product w.r.t. $L^{2}(\Gamma)$. This expression is integrated by part over $\Gamma$ to obtain the first part of the mixed formulation

$$
\mathrm{a}_{2}^{\mathrm{I}}\left(\tilde{e}^{\varepsilon, 2}, \tilde{\lambda}^{\varepsilon, 2} ; e^{\prime}\right)=\left\langle f, e^{\prime}\right\rangle \quad \forall e^{\prime} \in H_{\partial \Omega}^{1,1}(\Omega \backslash \Gamma)
$$

with

$$
\begin{aligned}
\mathrm{a}_{2}^{\mathrm{I}}\left(e, \lambda ; e^{\prime}\right)=\int_{\Omega \backslash \Gamma} \nabla e \cdot \nabla \overline{e^{\prime}} \mathrm{d} \underline{x}+\left(\mathrm{i} c_{0}-\varepsilon \frac{c_{0}^{2}}{6}-\varepsilon^{2} \frac{7 \mathrm{i} c_{0}^{3}}{240}\right)\left\langle\{e\},\left\{e^{\prime}\right\}\right\rangle_{\Gamma}+\varepsilon^{2} \frac{\mathrm{i} c_{0}}{12}\left\langle\partial_{t}\{e\}, \partial_{t}\left\{e^{\prime}\right\}\right\rangle_{\Gamma} & \\
& +\varepsilon^{2} \frac{\mathrm{i} c_{0}}{24}\left\langle\kappa \lambda,\left\{e^{\prime}\right\}\right\rangle_{\Gamma}+\left\langle\lambda,\left[e^{\prime}\right]\right\rangle_{\Gamma}
\end{aligned}
$$

and where we have introduced the test space $H_{\partial \Omega}^{1,1}(\Omega \backslash \Gamma)$ to ensure a minimal regularity on $\Gamma$

$$
H_{\partial \Omega}^{1,1}(\Omega \backslash \Gamma)=\left\{v \in H^{1}(\Omega \backslash \Gamma):\{v\} \in H^{1}(\Gamma), v=0 \text { on } \partial \Omega\right\} .
$$

The second part of the mixed formulation consists in a weak formulation of (5.1a)

$$
\mathrm{a}_{2}^{\mathrm{II}}\left(\tilde{e}_{\mathrm{ext}}^{\varepsilon, 2}, \tilde{\lambda}^{\varepsilon, 2} ; \lambda^{\prime}\right)=0, \quad \forall \lambda^{\prime} \in L^{2}(\Gamma)
$$

with

$$
\mathrm{a}_{2}^{\mathrm{II}}\left(e, \lambda ; \lambda^{\prime}\right)=\left\langle[e], \lambda^{\prime}\right\rangle_{\Gamma}+\varepsilon^{2} \frac{\mathrm{i} c_{0}}{24}\left\langle\{e\}, \kappa \lambda^{\prime}\right\rangle_{\Gamma}+\varepsilon^{2} \frac{\mathrm{i} c_{0}}{12}\left\langle\lambda, \lambda^{\prime}\right\rangle_{\Gamma} .
$$

The mixed formulation takes the form: Seek $\tilde{e}_{\text {ext }}^{\varepsilon, 2} \in H_{\partial \Omega}^{1,1}(\Omega \backslash \Gamma)$ and $\tilde{\lambda}_{\text {ext }}^{\varepsilon, 2} \in L^{2}(\Gamma)$ such that for all $e^{\prime} \in H_{\partial \Omega}^{1,1}(\Omega \backslash \Gamma)$ and $\lambda^{\prime} \in L^{2}(\Gamma)$

or equivalently

$$
\left\{\begin{array}{l}
\mathrm{a}_{2}^{\mathrm{I}}\left(\tilde{e}_{\mathrm{ext}}^{\varepsilon, 2}, \tilde{\lambda}_{\mathrm{ext}}^{\varepsilon, 2} ; e^{\prime}\right)=\left\langle f, e^{\prime}\right\rangle \\
\mathrm{a}_{2}^{\mathrm{II}}\left(\tilde{e}_{\mathrm{ext}}^{\varepsilon, 2}, \tilde{\lambda}_{\mathrm{ext}}^{\varepsilon, 2} ; \lambda^{\prime}\right)=0
\end{array}\right.
$$

$$
\mathrm{a}_{2}\left(\left(\begin{array}{c}
\tilde{e}_{\text {ext }}^{\varepsilon, 2} \\
\tilde{\lambda}_{\text {ext }}^{\varepsilon, 2}
\end{array}\right),\left(\begin{array}{c}
e^{\prime} \\
\lambda^{\prime}
\end{array}\right)\right)=\left\langle f, e^{\prime}\right\rangle
$$


with the continuous sesquilinear form on the product space $H_{\partial \Omega}^{1,1}(\Omega \backslash \Gamma) \times L^{2}(\Gamma)$

$$
\mathrm{a}_{2}\left(\left(\begin{array}{l}
e \\
\lambda
\end{array}\right),\left(\begin{array}{c}
e^{\prime} \\
\lambda^{\prime}
\end{array}\right)\right)=\mathrm{a}_{2}\left(e, \lambda ; e^{\prime}\right)+\mathrm{a}_{2}^{\mathbb{I}}\left(e, \lambda ; \lambda^{\prime}\right)
$$

Conversely, it is easy to check that solutions $\tilde{e}_{\text {ext }}^{\varepsilon, 2}$ and $\tilde{\lambda}_{\text {ext }}^{\varepsilon, 2}$ of (5.12) satisfy (3.6) and (5.5).

For the model of order 3 , this procedure can be as well repeated. This leads to a similar weak formulation where $a_{2}$ has to be replaced by $a_{3}$ given by

$$
\begin{aligned}
\mathrm{a}_{3}\left(\left(\begin{array}{c}
e \\
\lambda
\end{array}\right),\left(\begin{array}{c}
e^{\prime} \\
\lambda^{\prime}
\end{array}\right)\right)= & \mathrm{a}_{2}\left(\left(\begin{array}{c}
e \\
\lambda
\end{array}\right),\left(\begin{array}{c}
e^{\prime} \\
\lambda^{\prime}
\end{array}\right)\right)+\frac{17 c_{0}^{4} \varepsilon^{3}}{3360}\left\langle\{e\},\left\{e^{\prime}\right\}\right\rangle_{\Gamma}-\frac{c_{0}^{2} \varepsilon^{3}}{40}\left\langle\partial_{t}\{e\}, \partial_{t}\left\{e^{\prime}\right\}\right\rangle_{\Gamma} \\
& +\frac{c_{0}^{2} \varepsilon^{3}}{120}\left\langle\kappa\{e\}, \kappa\left\{e^{\prime}\right\}\right\rangle_{\Gamma}+\frac{c_{0}^{2} \varepsilon^{3}}{240}\left(\left\langle\kappa \lambda,\left\{e^{\prime}\right\}\right\rangle_{\Gamma}+\left\langle\{e\}, \kappa \lambda^{\prime}\right\rangle_{\Gamma}\right)+\frac{c_{0}^{2} \varepsilon^{3}}{120}\left\langle\lambda, \lambda^{\prime}\right\rangle_{\Gamma} .
\end{aligned}
$$

\subsection{Well-posedness and stability}

Even if the exact model provides a unique solution this does not transfer as a matter of course to the approximative models. In this section, we address the question of existence, uniqueness of the solutions and the stability with respect to the small parameter $\varepsilon$.

We start with a preliminary lemma.

Lemma 5.1 (equivalent $H_{\partial \Omega}^{1}(\Omega \backslash \Gamma)$-norm). Let $\alpha>0$. The semi-norm $\left(|v|_{H^{1}(\Omega \backslash \Gamma)}^{2}+\alpha\|\{v\}\|_{L^{2}(\Gamma)}^{2}\right)^{1 / 2}$ is a norm of $H_{\partial \Omega}^{1}(\Omega \backslash \Gamma)$.

Proof. We shall prove the equivalence to the standard $H^{1}(\Omega \backslash \Gamma)$-norm, i.e., there exists $0<\gamma_{-} \leq \gamma_{+}$such that

$$
\gamma_{-}\|v\|_{H^{1}(\Omega \backslash \Gamma)}^{2} \leq|v|_{H^{1}(\Omega \backslash \Gamma)}^{2}+\|\{v\}\|_{L^{2}(\Gamma)}^{2} \leq \gamma_{+}\|v\|_{H^{1}(\Omega \backslash \Gamma)}^{2} \quad \forall v \in H_{\partial \Omega}^{1}(\Omega \backslash \Gamma) .
$$

The second inequality follows from the the trace theorem on $\Gamma$. To prove the first one, we act by contradiction. Let us suppose that there exists a sequence $v_{n} \in H_{\partial \Omega}^{1}(\Omega \backslash \Gamma)$ satisfying

$$
\left\{\begin{array}{l}
\left\|v_{n}\right\|_{H^{1}(\Omega \backslash \Gamma)}=1, \\
\lim _{n \rightarrow+\infty}\left|v_{n}\right|_{H^{1}(\Omega \backslash \Gamma)}^{2}=0 \\
\lim _{n \rightarrow+\infty}\left\|\left\{v_{n}\right\}\right\|_{L^{2}(\Gamma)}^{2}=0 .
\end{array}\right.
$$

One can extract from $v_{n}$ a subsequence $w_{n}$ weakly convergent in $H^{1}(\Omega \backslash \Gamma)$ to its limit $w$ which satisfies

$$
\nabla w=0 \text { in } \Omega \backslash \Gamma, \quad w=0 \text { on } \partial \Omega \quad \text { and } \quad\{w\}=0 \text { on } \Gamma .
$$

Let us prove that $w=0$. Since $\nabla w=0$ in $\Omega \backslash \Gamma$, the function $w$ is constant in the two connected subdomains of $\Omega \backslash \Gamma$. Moreover, these two constants are zero since $w=0$ on $\partial \Omega$ and $\{w\}=0$ on $\Gamma$. Hence, we can conclude that $w_{n}$ is weakly convergent in $H^{1}(\Omega \backslash \Gamma)$ to 0 , and by compactness, strongly convergent in $L^{2}(\Omega \backslash \Gamma)$. Consequently, the function $w_{n}$ satisfies

$$
\left\{\begin{array}{l}
\left\|w_{n}\right\|_{H^{1}(\Omega \backslash \Gamma)}=1, \\
\lim _{n \rightarrow+\infty}\left|w_{n}\right|_{H^{1}(\Omega \backslash \Gamma)}^{2}=0, \\
\lim _{n \rightarrow+\infty}\left\|w_{n}\right\|_{L^{2}(\Omega \backslash \Gamma)}^{2}=0 .
\end{array}\right.
$$

This is impossible. 
Theorem 5.2 (well-posedness and stability of the models of order 2). There exists $\varepsilon^{*}>0$ such that for all $\left.\varepsilon \in] 0, \varepsilon^{*}\right], f \in\left(H_{\partial \Omega}^{1,1}(\Omega \backslash \Gamma)\right)^{\prime}$ and $g \in L^{2}(\Gamma)$ there is a unique $(e, \lambda) \in H_{\partial \Omega}^{1,1}(\Omega \backslash \Gamma) \times L^{2}(\Gamma)$ satisfying

$$
\left\langle f, e^{\prime}\right\rangle+\left\langle g, \lambda^{\prime}\right\rangle_{\Gamma}=\mathrm{a}_{2}\left(\left(\begin{array}{c}
e \\
\lambda
\end{array}\right),\left(\begin{array}{c}
e^{\prime} \\
\lambda^{\prime}
\end{array}\right)\right), \quad \forall\left(e^{\prime}, \lambda^{\prime}\right) \in H_{\partial \Omega}^{1,1}(\Omega \backslash \Gamma) \times L^{2}(\Gamma) .
$$

Moreover, there exists a constant $C>0$ independent of $\left.\varepsilon \in] 0, \varepsilon^{*}\right], f \in\left(H_{\partial \Omega}^{1}(\Omega \backslash \Gamma)\right)^{\prime}$ and $g \in L^{2}(\Gamma)$ such that

$$
\|e\|_{H_{\partial \Omega}^{1}(\Omega \backslash \Gamma)}^{2}+\varepsilon^{2}|\{e\}|_{H^{1}(\Gamma)}^{2}+\varepsilon^{2}\|\lambda\|_{L^{2}(\Gamma)}^{2} \leq C\left(\|f\|_{\left(H_{\partial \Omega}^{1}(\Omega \backslash \Gamma)\right)^{\prime}}^{2}+\varepsilon^{-2}\|g\|_{L^{2}(\Gamma)}^{2}\right) .
$$

Proof. We pick $\varepsilon^{*}=\min \left(\frac{1}{c_{0}}, \frac{1}{\|\kappa\|_{L^{\infty}(\Gamma)}}\right)$, and consider therefore in the continuation, $\varepsilon>0$ satisfying $\varepsilon c_{0} \leq 1$ and $\varepsilon\|\kappa\|_{L^{\infty}(\Gamma)} \leq 1$.

Operator formulation.. Let us first associate to the bilinear form $\mathrm{a}_{2}$, a linear operator $A_{2}$

$$
A_{2}: H_{\partial \Omega}^{1,1}(\Omega \backslash \Gamma) \times L^{2}(\Gamma) \longrightarrow\left(H_{\partial \Omega}^{1,1}(\Omega \backslash \Gamma)\right)^{\prime} \times L^{2}(\Gamma) \quad(e, \lambda) \longmapsto(f, g)
$$

defined by

$$
\left\langle f, e^{\prime}\right\rangle+\left\langle g, \lambda^{\prime}\right\rangle_{\Gamma}=\mathrm{a}_{2}\left(\left(\begin{array}{l}
e \\
\lambda
\end{array}\right),\left(\begin{array}{c}
e^{\prime} \\
\lambda^{\prime}
\end{array}\right)\right), \quad \forall\left(e^{\prime}, \lambda^{\prime}\right) \in H_{\partial \Omega}^{1,1}(\Omega \backslash \Gamma) \times L^{2}(\Gamma)
$$

or equivalently

$$
\begin{aligned}
& \left\langle f, e^{\prime}\right\rangle=\mathrm{a}_{2}^{\mathrm{I}}\left(e, \lambda ; e^{\prime}\right), \quad \forall e^{\prime} \in H_{\partial \Omega}^{1,1}(\Omega \backslash \Gamma) \\
& \left\langle g, \lambda^{\prime}\right\rangle_{\Gamma}=\mathrm{a}_{2}^{\mathrm{I}}\left(e, \lambda ; \lambda^{\prime}\right), \quad \forall \lambda^{\prime} \in L^{2}(\Gamma) .
\end{aligned}
$$

To prove that the problem of order 2 is well-posed, we will show that $A_{2}$ is bijective. First, we prove that $A_{2}$ is injective and has closed range, then we prove that the range of $A_{2}$ is dense in $\left(H_{\partial \Omega}^{1,1}(\Omega \backslash \Gamma)\right)^{\prime} \times L^{2}(\Gamma)$.

Injectivity and closed range. To show that the operator $A_{2}$ is injective and has closed range it suffices to prove the stability estimate (5.20) for every $(e, \lambda) \in H_{\partial \Omega}^{1,1}(\Omega \backslash \Gamma) \times L^{2}(\Gamma)$ and $(f, g)=A_{2}(e, \lambda)$. For proving surjectivity we consider here the range with $f \in\left(H_{\partial \Omega}^{1,1}(\Omega \backslash \Gamma)\right)^{\prime}$, i.e., in a larger space than the $f$ in the statement of the theorem. Let us equip the space $H_{\partial \Omega}^{1,1}(\Omega \backslash \Gamma)$ with the norm defined by

$$
\|u\|_{H^{1,1}(\Omega \backslash \Gamma)}^{2}:=\|u\|_{H^{1}(\Omega \backslash \Gamma)}^{2}+\varepsilon^{2}|\{u\}|_{H^{1}(\Gamma)}^{2} .
$$

By definition the functions $f$ and $g$ satisfy for every $\left(e^{\prime}, \lambda^{\prime}\right) \in H_{\partial \Omega}^{1,1}(\Omega \backslash \Gamma) \times L^{2}(\Gamma)$

$$
\begin{aligned}
\int_{\Omega \backslash \Gamma} \nabla e \cdot \nabla \overline{e^{\prime}} \mathrm{d} \underline{x}+ & \left(\mathrm{i} c_{0}-\varepsilon \frac{c_{0}^{2}}{6}-\varepsilon^{2} \frac{7 \mathrm{i} c_{0}^{3}}{240}\right)\left\langle\{e\},\left\{e^{\prime}\right\}\right\rangle_{\Gamma} \\
+ & \varepsilon^{2} \frac{\mathrm{i} c_{0}}{12}\left\langle\partial_{t}\{e\}, \partial_{t}\left\{e^{\prime}\right\}\right\rangle_{\Gamma}+\varepsilon^{2} \frac{\mathrm{i} c_{0}}{24}\left\langle\kappa \lambda,\left\{e^{\prime}\right\}\right\rangle_{\Gamma}+\left\langle\lambda,\left[e^{\prime}\right]\right\rangle_{\Gamma}=\left\langle f, e^{\prime}\right\rangle, \\
& \left\langle[e], \lambda^{\prime}\right\rangle_{\Gamma}+\varepsilon^{2} \frac{\mathrm{i} c_{0}}{24}\left\langle\{e\}, \kappa \lambda^{\prime}\right\rangle_{\Gamma}+\varepsilon^{2} \frac{\mathrm{i} c_{0}}{12}\left\langle\lambda, \lambda^{\prime}\right\rangle_{\Gamma}=\left\langle g, \lambda^{\prime}\right\rangle_{\Gamma} .
\end{aligned}
$$


Subtracting for $e^{\prime}=e$ and $\lambda^{\prime}=\lambda$ the conjugate complex of the second equation from the first equation and taking the real and imaginary parts we have

$$
\begin{aligned}
\operatorname{Re}\left(\langle f, e\rangle-\overline{\langle g, \lambda\rangle_{\Gamma}}\right) & =\operatorname{Re}\left(\mathrm{a}_{2}^{\mathrm{I}}(e, \lambda ; e)-\overline{\mathrm{a}_{2}^{\mathrm{II}}(e, \lambda ; \lambda)}\right)=|e|_{H^{1}(\Omega \backslash \Gamma)}^{2}-\varepsilon \frac{c_{0}^{2}}{6}\|\{e\}\|_{L^{2}(\Gamma)}^{2}-\varepsilon^{2} \frac{c_{0}}{12} \operatorname{Im}\left(\langle\kappa(t) \lambda,\{e\}\rangle_{\Gamma}\right), \\
\operatorname{Im}\left(\langle f, e\rangle-\overline{\langle g, \lambda\rangle_{\Gamma}}\right) & =\operatorname{Im}\left(\mathrm{a}_{2}^{\mathrm{I}}(e, \lambda ; e)-\overline{\mathrm{a}_{2}^{\mathrm{II}}(e, \lambda ; \lambda)}\right) \\
& =c_{0}\left(1-\varepsilon^{2} \frac{7 c_{0}^{2}}{240}\right)\|\{e\}\|_{L^{2}(\Gamma)}^{2}+\varepsilon^{2} \frac{c_{0}}{12}|\{e\}|_{H^{1}(\Gamma)}^{2}+\varepsilon^{2} \frac{c_{0}}{12} \operatorname{Re}\left(\langle\kappa(t) \lambda,\{e\}\rangle_{\Gamma}\right)+\varepsilon^{2} \frac{c_{0}}{12}\|\lambda\|_{L^{2}(\Gamma)}^{2} .
\end{aligned}
$$

Due to Young's inequality we can assert that

$$
\left|\langle\kappa(t) \lambda,\{e\}\rangle_{\Gamma}\right| \leq\|\kappa\|_{L^{\infty}(\Gamma)}\|\lambda\|_{L^{2}(\Gamma)}\|\{e\}\|_{L^{2}(\Gamma)} \leq \frac{\|\lambda\|_{L^{2}(\Gamma)}^{2}}{4}+\|\kappa\|_{L^{\infty}(\Gamma)}^{2}\|\{e\}\|_{L^{2}(\Gamma)}^{2}
$$

and consequently

$$
\begin{aligned}
& \operatorname{Re}\left(\langle f, e\rangle-\overline{\langle g, \lambda\rangle_{\Gamma}}\right) \geq|e|_{H^{1}(\Omega \backslash \Gamma)}^{2}-c_{0}\left(\frac{\varepsilon c_{0}}{6}+\frac{\varepsilon^{2}\|\kappa\|_{L^{\infty}(\Gamma)}^{2}}{12}\right)\|\{e\}\|_{L^{2}(\Gamma)}^{2}-\varepsilon^{2} \frac{c_{0}}{48}\|\lambda\|_{L^{2}(\Gamma)}^{2}, \\
& \operatorname{Im}\left(\langle f, e\rangle-\overline{\langle g, \lambda\rangle_{\Gamma}}\right) \geq c_{0}\left(1-\frac{7 \varepsilon^{2} c_{0}^{2}}{240}-\frac{\varepsilon^{2}\|\kappa\|_{L^{\infty}(\Gamma)}^{2}}{12}\right)\|\{e\}\|_{L^{2}(\Gamma)}^{2}+\frac{c_{0} \varepsilon^{2}}{12}|\{e\}|_{H^{1}(\Gamma)}^{2}+\varepsilon^{2} \frac{3 c_{0}}{48}\|\lambda\|_{L^{2}(\Gamma)}^{2} .
\end{aligned}
$$

Using the assumptions $\varepsilon\|\kappa\|_{L^{\infty}(\Gamma)} \leq 1$ and $\varepsilon c_{0} \leq 1$ we obtain

$$
\begin{aligned}
& \operatorname{Re}\left(\langle f, e\rangle-\overline{\langle g, \lambda\rangle_{\Gamma}}\right) \geq|e|_{H^{1}(\Omega \backslash \Gamma)}^{2}-\frac{c_{0}}{4}\|\{e\}\|_{L^{2}(\Gamma)}^{2}-\varepsilon^{2} \frac{c_{0}}{48}\|\lambda\|_{L^{2}(\Gamma)}^{2}, \\
& \operatorname{Im}\left(\langle f, e\rangle-\overline{\langle g, \lambda\rangle_{\Gamma}}\right) \geq \frac{3 c_{0}}{4}\|\{e\}\|_{L^{2}(\Gamma)}^{2}+\frac{c_{0} \varepsilon^{2}}{12}|\{e\}|_{H^{1}(\Gamma)}^{2}+\varepsilon^{2} \frac{3 c_{0}}{48}\|\lambda\|_{L^{2}(\Gamma)}^{2} .
\end{aligned}
$$

Remarking that $\left.\operatorname{Re}\left((1-\mathrm{i})\left(\langle f, e\rangle-\overline{\langle g, \lambda\rangle_{\Gamma}}\right)\right)=\operatorname{Re}\left(\langle f, e\rangle-\overline{\langle g, \lambda\rangle_{\Gamma}}\right)+\operatorname{Im}\left(\langle f, e\rangle-\overline{\langle g, \lambda\rangle_{\Gamma}}\right)\right)$ we obtain by adding the two lines of $(5.27)$

$$
\operatorname{Re}\left((1-\mathrm{i})\left(\langle f, e\rangle-\overline{\langle g, \lambda\rangle_{\Gamma}}\right)\right) \geq|e|_{H^{1}(\Omega \backslash \Gamma)}^{2}+\frac{c_{0}}{2}\|\{e\}\|_{L^{2}(\Gamma)}^{2}+\varepsilon^{2} \frac{c_{0}}{12}|\{e\}|_{H^{1}(\Gamma)}^{2}+\varepsilon^{2} \frac{c_{0}}{24}\|\lambda\|_{L^{2}(\Gamma)}^{2} .
$$

Due to the Poincaré-Friedrichs inequality, see Lemma 5.1, $|e|_{H^{1}(\Omega \backslash \Gamma)}^{2}+\|\{e\}\|_{L^{2}(\Gamma)}^{2} \geq \gamma\|e\|_{H^{1}(\Omega \backslash \Gamma)}^{2}$ we get the estimate

$$
\operatorname{Re}\left((1-\mathrm{i})\left(\langle f, e\rangle-\overline{\langle g, \lambda\rangle_{\Gamma}}\right)\right) \geq C_{1}\left(\|e\|_{H^{1}(\Omega \backslash \Gamma)}^{2}+\varepsilon^{2}|\{e\}|_{H^{1}(\Gamma)}^{2}+\varepsilon^{2}\|\lambda\|_{L^{2}(\Gamma)}\right)
$$

with $C_{1}>0$ independent of $\varepsilon$. The Cauchy-Schwartz inequality allows to bound the left hand-side

$$
\begin{aligned}
\operatorname{Re}\left((1-\mathrm{i})\left(\langle f, e\rangle-\overline{\langle g, \lambda\rangle_{\Gamma}}\right)\right) & \leq \sqrt{2}\left(\|f\|_{\left(H^{1,1}(\Omega \backslash \Gamma)\right)^{\prime}}\|e\|_{H^{1,1}(\Omega \backslash \Gamma)}+\varepsilon^{-1}\|g\|_{L^{2}(\Gamma)} \varepsilon\|\lambda\|_{L^{2}(\Gamma)}\right) \\
& \leq \sqrt{2}\left(\|f\|_{\left(H^{1,1}(\Omega \backslash \Gamma)\right)^{\prime}}^{2}+\varepsilon^{-2}\|g\|_{L^{2}(\Gamma)}^{2}\right)^{\frac{1}{2}}\left(\|e\|_{H^{1,1}(\Omega \backslash \Gamma)}^{2}+\varepsilon^{2}\|\lambda\|_{L^{2}(\Gamma)}^{2}\right)^{\frac{1}{2}}
\end{aligned}
$$

and using (5.29) we get

$$
\left(\|e\|_{H^{1,1}(\Omega \backslash \Gamma)}^{2}+\varepsilon^{2}\|\lambda\|_{L^{2}(\Gamma)}^{2}\right) \leq C_{2}\left(\|f\|_{\left(H^{1,1}(\Omega \backslash \Gamma)\right)^{\prime}}^{2}+\varepsilon^{-2}\|g\|_{L^{2}(\Gamma)}^{2}\right)^{\frac{1}{2}}\left(\|e\|_{H^{1,1}(\Omega \backslash \Gamma)}^{2}+\varepsilon^{2}\|\lambda\|_{L^{2}(\Gamma)}^{2}\right)^{\frac{1}{2}}
$$

which proves the injectivity and closedness of the range. With $\|f\|_{\left(H^{1,1}(\Omega \backslash \Gamma)\right)^{\prime}} \leq\|f\|_{\left(H^{1}(\Omega \backslash \Gamma)\right)^{\prime}}$ the stability statement (5.20) holds with a constant independant of $\varepsilon$. 
Density of the range.. Let us prove that every $\left(e^{\prime}, \lambda^{\prime}\right) \in H_{\partial \Omega}^{1,1}(\Omega \backslash \Gamma) \times L^{2}(\Gamma)$ in the orthogonal complement of the range of $A_{2}$, i.e.,

$$
0=\mathrm{a}_{2}\left(\left(\begin{array}{l}
e \\
\lambda
\end{array}\right),\left(\begin{array}{c}
e^{\prime} \\
\lambda^{\prime}
\end{array}\right)\right) \quad \forall(e, \lambda) \in H_{\partial \Omega}^{1,1}(\Omega \backslash \Gamma) \times L^{2}(\Gamma)
$$

satisfy $e^{\prime}=0$ and $\lambda^{\prime}=0$ (see for example [7], Corollary 1.8).

We first consider $e=e^{\prime}$ and $\lambda=0$ which leads to

$$
\begin{aligned}
0 & =\mathrm{a}_{2}\left(\left(\begin{array}{c}
e^{\prime} \\
0
\end{array}\right),\left(\begin{array}{c}
e^{\prime} \\
\lambda^{\prime}
\end{array}\right)\right)=: \mathrm{b}_{2}^{\mathrm{I}}\left(e^{\prime} ; e^{\prime}, \lambda^{\prime}\right) \\
& =\left|e^{\prime}\right|_{H^{1}(\Omega \backslash \Gamma)}^{2}+\left(\mathrm{i} c_{0}-\frac{c_{0}^{2} \varepsilon}{6}-\frac{7 \mathrm{i} c_{0}^{3} \varepsilon^{2}}{240}\right)\left\|\left\{e^{\prime}\right\}\right\|_{L^{2}(\Gamma)}^{2}+\frac{\mathrm{i} c_{0} \varepsilon^{2}}{12}\left|\left\{e^{\prime}\right\}\right|_{H^{1}(\Gamma)}^{2}+\left\langle\left[e^{\prime}\right], \lambda^{\prime}\right\rangle_{\Gamma}+\frac{\mathrm{i} c_{0} \varepsilon^{2}}{24}\left\langle\left\{e^{\prime}\right\}, \kappa \lambda^{\prime}\right\rangle_{\Gamma} .
\end{aligned}
$$

Then, we have for $e=0$ and $\lambda=\lambda^{\prime}$

$$
0=\mathrm{a}_{2}\left(\left(\begin{array}{c}
0 \\
\lambda^{\prime}
\end{array}\right),\left(\begin{array}{c}
e^{\prime} \\
\lambda^{\prime}
\end{array}\right)\right)=: \mathrm{b}_{2}^{\mathbb{I}}\left(\lambda^{\prime} ; e^{\prime}, \lambda^{\prime}\right)=\frac{\mathrm{i} c_{0} \varepsilon^{2}}{24}\left\langle\kappa \lambda^{\prime},\left\{e^{\prime}\right\}\right\rangle_{\Gamma}+\left\langle\lambda^{\prime},\left[e^{\prime}\right]\right\rangle_{\Gamma}+\frac{\mathrm{i} c_{0} \varepsilon^{2}}{12}\left\langle\lambda^{\prime}, \lambda^{\prime}\right\rangle_{\Gamma} .
$$

In general, it holds $\mathrm{b}_{2}^{\mathrm{I}}\left(e^{\prime} ; e^{\prime}, \lambda^{\prime}\right) \neq \mathrm{a}_{2}^{\mathrm{I}}\left(e^{\prime}, \lambda^{\prime} ; e^{\prime}\right)$ as well as $\mathrm{b}_{2}^{\mathbb{I}}\left(\lambda^{\prime} ; e^{\prime}, \lambda\right)^{\prime} \neq \mathrm{a}_{2}^{\mathbb{I}}\left(\lambda^{\prime}, e^{\prime} ; \lambda^{\prime}\right)$. However, it is easy to verify that

$$
0=\mathrm{b}_{2}^{\mathrm{I}}\left(e^{\prime} ; e^{\prime}, \lambda^{\prime}\right)-\overline{\mathrm{b}_{2}^{\mathrm{II}}\left(\lambda^{\prime} ; e^{\prime}, \lambda^{\prime}\right)}=\mathrm{a}_{2}^{\mathrm{I}}\left(e^{\prime}, \lambda^{\prime} ; e^{\prime}\right)-\overline{\mathrm{a}_{2}^{\mathrm{II}}\left(e^{\prime}, \lambda^{\prime} ; \lambda^{\prime}\right)} .
$$

Thus, it holds (5.24) with $e^{\prime}$ instead of $e, \lambda^{\prime}$ instead of $\lambda$ and $f=g=0$. Thus, repeating the steps in the first part of the proof leads to

$$
e^{\prime}=0 \quad \text { in } \Omega \backslash \Gamma, \quad \text { and } \quad \lambda^{\prime}=0 \quad \text { on } \Gamma,
$$

and so the range of $A_{2}$ is dense, which completes the proof.

Remark 5.3. The upper bound for thicknesses $\varepsilon^{*}$ in the proof for stability was not choosen optimally. For particular sheets with a certain $c_{0}$ existence and uniqueness might hold for larger thicknesses.

Remark 5.4. The same well-posed and stability result can be stated as well for the model of order 3 .

\subsection{Estimates of the modelling error}

With the stability estimate we may deduce the modelling error induced by the neglected terms in their derivation. These neglected terms are of order $\varepsilon^{N+1}$. Due to the $\varepsilon^{-2}$-term in front of the norm of $g$ in the stability estimate (5.20) we can only obtain a non-optimal estimate of the modelling error of order $\varepsilon^{N}$. However, using an asymptotic expansion of $\tilde{e}_{\text {ext }}^{\varepsilon, N}$ up to one order higher we will be able to prove the following theorem.

Theorem 5.5 (modelling error). Let $N=2,3$. We have the estimate

$$
\left\|\tilde{e}_{\mathrm{ext}}^{\varepsilon, N}-e_{\mathrm{ext}}^{\varepsilon}\right\|_{H^{1}\left(\Omega_{\mathrm{ext}}^{\varepsilon}\right)}=\underset{\varepsilon \rightarrow 0}{O}\left(\varepsilon^{N+1}\right) .
$$

Proof. To obtain an error estimate with the optimal order $O\left(\varepsilon^{N+1}\right)$ we will first derive asymptotic expansions

$$
\tilde{e}_{\mathrm{ext}}^{\varepsilon, N}, m \sum_{j=0}^{m} \varepsilon^{j} v_{\mathrm{ext}}^{N, j}
$$

of the solutions $\tilde{e}_{\mathrm{ext}}^{\varepsilon, N}$, prove then that $\tilde{e}_{\mathrm{ext}}^{\varepsilon, N, m}$ approximate $\tilde{e}_{\mathrm{ext}}^{\varepsilon, N}$ to order $\varepsilon^{m+1}$, and conclude with the fact that the expansion (5.34) coincides up to order $m \leq N$ with the asymptotic expansion of the exact solution. 
Step 1.. Let $N=2$ or 3. We insert the asymptotic expansion (5.34) in (3.6) and identify order by order. The functions $v_{\mathrm{ext}}^{N, j}$ satisfies

$$
\begin{aligned}
& v_{\mathrm{ext}}^{N, j} \in H_{\Delta}^{1}(\Omega \backslash \Gamma), \\
&-\Delta v_{\mathrm{ext}}^{N, j}(\underline{x})=f_{j}(\underline{x}), \quad \text { in } \Omega \backslash \Gamma, \\
& {\left[v_{\mathrm{ext}}^{N, j}\right](t) }=\sum_{\ell=2}^{N}\left(\gamma_{\ell} v_{\mathrm{ext}}^{N, j-\ell}\right)(t), \quad \text { on } \Gamma, \\
& {\left[\partial_{n} v_{\mathrm{ext}}^{N, j}\right](t)-\mathrm{i} c_{0}\left\{v_{\mathrm{ext}}^{N, j}\right\}(t)=\sum_{\ell=1}^{N}\left(\boldsymbol{\zeta}_{\ell} v_{\mathrm{ext}}^{N, j-\ell}\right)(t), \quad \text { on } \Gamma, }
\end{aligned}
$$

completed with the source terms

$$
f_{0}(\underline{x})=f(\underline{x}) \quad \text { and } \quad f_{j}(\underline{x})=0, \quad \text { in } \Omega \backslash \Gamma
$$

and the convention $v_{\mathrm{ext}}^{N, j}=0$ for $j<0$.

The terms $v_{\mathrm{ext}}^{N, j}$ of these asymptotic expansions do not depend on $\varepsilon$ and are well defined. These functions are in general discontinuous across $\Gamma$ but are $C^{\infty}\left(\Omega_{+}\right)$and $C^{\infty}\left(\Omega_{-}\right)$(see Appendix B for a proof of this regularity result).

Step 2. To show that the expansion (5.34) is in fact an approximation of $\tilde{e}_{\text {ext }}^{\varepsilon, N}$ we define the residual

$$
r_{\mathrm{ext}}^{\varepsilon, N}:=\tilde{e}_{\mathrm{ext}}^{\varepsilon, N}-\sum_{j=0}^{m} \varepsilon^{j} v_{\mathrm{ext}}^{N, j}
$$

and we call

$$
\rho_{\mathrm{ext}}^{\varepsilon, N, m}:=\left\{\partial_{n} r_{\mathrm{ext}}^{\varepsilon, N, m}\right\}=\tilde{\lambda}_{\mathrm{ext}}^{\varepsilon, N}-\sum_{j=0}^{m} \varepsilon^{j}\left\{\partial_{n} v_{\mathrm{ext}}^{N, j}\right\} .
$$

The residual $r_{\mathrm{ext}}^{\varepsilon, N} \in H_{\partial \Omega}^{1,1}(\Omega \backslash \Gamma)$ satisfies

$$
\left\{\begin{array}{rlrl}
-\Delta r_{\mathrm{ext}}^{\varepsilon, N, m} & =0 & & \text { in } \Omega \backslash \Gamma, \\
{\left[r_{\mathrm{ext}}^{\varepsilon, N, m}\right](t)-\sum_{\ell=2}^{N}\left(\varepsilon^{\ell} \boldsymbol{\gamma}_{\ell} r_{\mathrm{ext}}^{\varepsilon, N, m}\right)(t)=f^{\varepsilon, N, m}(t),} & & \text { on } \Gamma \\
{\left[\partial_{n} r_{\mathrm{ext}}^{\varepsilon, N, m}\right](t)-\mathrm{i} c_{0}\left\{r_{\mathrm{ext}}^{\varepsilon, N, m}\right\}(t)-\sum_{\ell=1}^{N}\left(\varepsilon^{\ell} \boldsymbol{\zeta}_{\ell} r_{\mathrm{ext}}^{\varepsilon, N, m}\right)(t)} & =g^{\varepsilon, N, m}(t), & & \text { on } \Gamma \\
r_{\mathrm{ext}}^{\varepsilon, N} & =0, & & \text { on } \partial \Omega,
\end{array}\right.
$$

with

$$
f^{\varepsilon, N, m}(t)=-\sum_{(j, \ell) \in \mathrm{S}_{N, m}} \varepsilon^{j+\ell}\left(\gamma_{\ell} v_{\mathrm{ext}}^{N, j}\right)(t), \quad g^{\varepsilon, N, m}(t)=-\sum_{(j, \ell) \in \mathrm{S}_{N, m}} \varepsilon^{j+\ell}\left(\boldsymbol{\zeta}_{\ell} v_{\mathrm{ext}}^{N, j}\right)(t)
$$


where the domain of summation $S_{n, m}$ is the set of integers

$$
\mathrm{S}_{N, m}=\left\{(j, \ell) \in \mathbb{N}^{2}: j \in \llbracket 0, m \rrbracket, \quad \ell \in \llbracket 0, N \rrbracket \quad \text { and } j+\ell \geq m+1\right\} .
$$

The system (5.38) can be transformed into a variational problem

$$
\mathrm{a}_{N}\left(\left(\begin{array}{c}
r_{\mathrm{ext}}^{\varepsilon, N, m} \\
\rho_{\text {ext }}^{\varepsilon, N, m}
\end{array}\right),\left(\begin{array}{c}
e^{\prime} \\
\lambda^{\prime}
\end{array}\right)\right)=\left\langle g^{\varepsilon, N, m},\left\{e^{\prime}\right\}\right\rangle_{\Gamma}+\left\langle f^{\varepsilon, N, m}, \lambda^{\prime}\right\rangle_{\Gamma}, \quad \forall e^{\prime} \in H_{\partial \Omega}^{1,1}(\Omega \backslash \Gamma), \forall \lambda^{\prime} \in L^{2}(\Gamma) .
$$

Since the functions $v_{\text {ext }}^{N, m}$ do not depend on $\varepsilon$ and are regular, we have the bounds

$$
\left\|\gamma_{\ell} v_{\mathrm{ext}}^{N, j}\right\|_{L^{2}(\Gamma)} \leq C_{N, j} \quad \text { and } \quad\left\|\zeta_{\ell v_{\mathrm{ext}}^{N, j}}\right\|_{L^{2}(\Gamma)} \leq C_{N, j}^{\prime}
$$

These two sums contain a finite number of monomials of $\varepsilon$ with order larger than $m+1$.

$$
\left\|f^{\varepsilon, N, m}\right\|_{L^{2}(\Gamma)}=\underset{\varepsilon \rightarrow 0}{O}\left(\varepsilon^{m+1}\right) \quad \text { and } \quad\left\|g^{\varepsilon, N, m}\right\|_{L^{2}(\Gamma)}=\underset{\varepsilon \rightarrow 0}{O}\left(\varepsilon^{m+1}\right)
$$

Applying Theorem 5.2 allows to obtain a non-optimal bound $\left\|r_{\mathrm{ext}}^{\varepsilon, N, m}\right\|_{H^{1}(\Omega \backslash \Gamma)} \leq\left\|r_{\mathrm{ext}}^{\varepsilon, N, m}\right\|_{H^{1,1}(\Omega \backslash \Gamma)}=O\left(\varepsilon^{m}\right)$. The optimal estimates follow from a triangular inequality

$$
\left\|r_{\mathrm{ext}}^{\varepsilon, N, m}\right\|_{H^{1}(\Omega \backslash \Gamma)} \leq\left\|r_{\mathrm{ext}}^{\varepsilon, N, m+1}\right\|_{H^{1}(\Omega \backslash \Gamma)}+\varepsilon^{m+1}\left\|v_{\mathrm{ext}}^{N, m+1}\right\|_{H^{1}(\Omega \backslash \Gamma)}=\underset{\varepsilon \rightarrow 0}{O}\left(\varepsilon^{m+1}\right) .
$$

Step 3.. Comparing the systems (2.5) and (5.35), we remark that $v_{\text {ext }}^{N, j}=u_{\text {ext }}^{j}$ for $j \in \llbracket 0, N \rrbracket$ (they are different for $j>N)$. Inserting $e_{\text {ext }}^{\varepsilon, N}=\sum_{j=0}^{N} \varepsilon^{j} u_{\text {ext }}^{j}=\sum_{j=0}^{N} \varepsilon^{j} v_{\text {ext }}^{N, j}$, we get by the triangular inequality since $\Omega_{\text {ext }}^{\varepsilon} \subset \Omega \backslash \Gamma$

$$
\left\|\tilde{e}_{\mathrm{ext}}^{\varepsilon, N}-e_{\mathrm{ext}}^{\varepsilon}\right\|_{H^{1}\left(\Omega_{\mathrm{ext}}^{\varepsilon}\right)} \leq\left\|\tilde{e}_{\mathrm{ext}}^{\varepsilon, N}-e_{\mathrm{ext}}^{\varepsilon, N}\right\|_{H^{1}(\Omega \backslash \Gamma)}+\left\|e_{\mathrm{ext}}^{\varepsilon, N}-e_{\mathrm{ext}}^{\varepsilon}\right\|_{H^{1}\left(\Omega_{\mathrm{ext}}^{\varepsilon}\right)}
$$

with $r_{\mathrm{ext}}^{\varepsilon, N}=e_{\mathrm{ext}}^{\varepsilon}-e_{\mathrm{ext}}^{\varepsilon, N}$ and $r_{\mathrm{ext}}^{\varepsilon, N, N}=\tilde{e}_{\mathrm{ext}}^{\varepsilon, N}-e_{\mathrm{ext}}^{\varepsilon, N}$ the two estimates (2.3) and (5.42) lead to the conclusion

$$
\left\|\tilde{e}_{\mathrm{ext}}^{\varepsilon, N}-e_{\mathrm{ext}}^{\varepsilon}\right\|_{H^{1}\left(\Omega_{\mathrm{ext}}^{\varepsilon}\right)}=\underset{\varepsilon \rightarrow 0}{O}\left(\varepsilon^{N+1}\right) .
$$

\section{NumERICAL EXAMPLES}

This section is devoted to the numerical validation of the approximate models of order 1,2 and 3 . The simulations were performed with the numerical $\mathrm{C}++$ library Concepts $[9,11]$ using exactly curved elements of high order which permits discretisation error lying below the modelling error.

The geometrical setting of the experiments. We consider an ellipsoidal thin sheet, a sheet with varying curvature, with two live circular conductors in the middle (with opposite direction of the currents). The problem is completed by perfect magnetic conductor (PMC) boundary condition on the circular outer boundary which turns out to be a homogeneous Neumann boundary condition. Note, that the proof for stability and the modelling error of the approximative problems with Dirichlet boundary condition can be extended to the case of Neumann boundary condition using $H^{1}(\Omega \backslash \Gamma) \backslash \mathbb{C}$ instead of $H_{\partial \Omega}^{1}(\Omega \backslash \Gamma)$ (see Lem. 5.1). See Figure 2a for a sketch of the geometry and Figure $2 \mathrm{~b}$ for the flux lines and the absolute value of the magnetic field induced by the two wires and shielded by the thin sheet (computed with the exact model). 

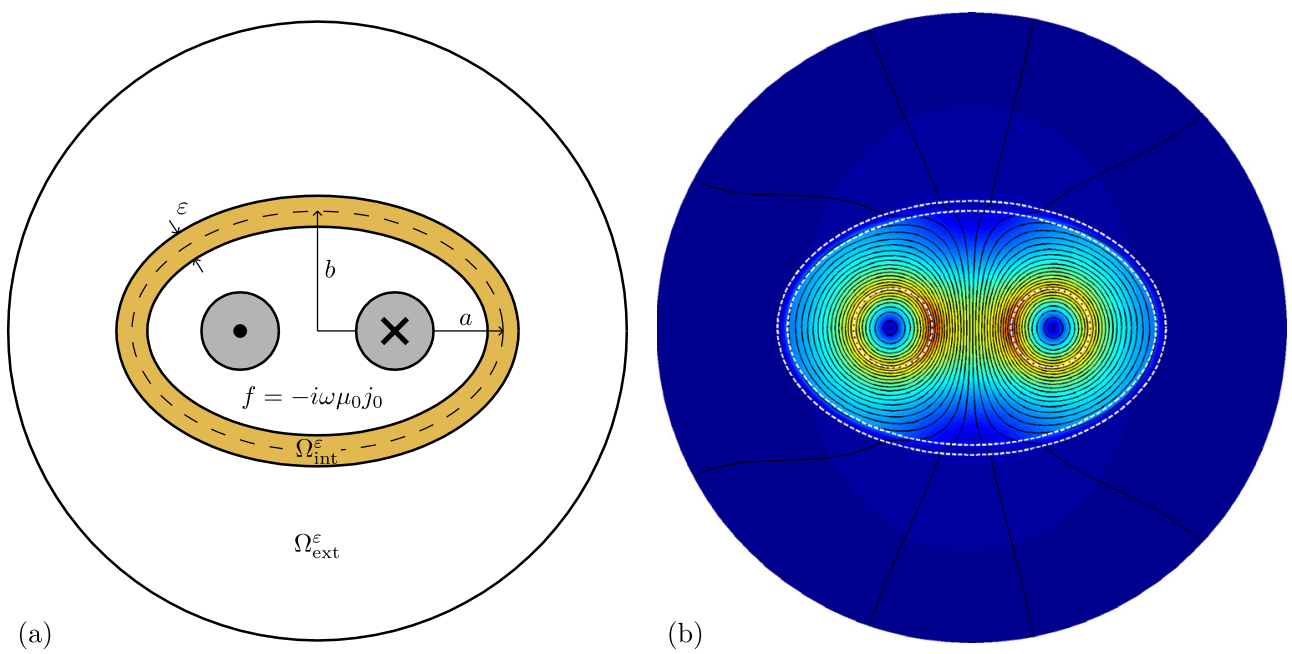

Figure 2. (a) Geometrical setting with elliptic mid-line (dashed line) with the semi-major axis $a=1.2$ and semi-minor axis $b=\sqrt{0.6}$. The boundary is a circle of radius $R=2$. The live wires are circles of radius 0.25 and midpoints $( \pm 0.5,0)$. (b) The magnitude and the flux lines of the in-plane magnetic field for $\varepsilon=1 / 16, c_{0}=10$ and $f=1$ in the left wire and $f=-1$ in the right one - corresponding to an alternating currents $j_{0}$ with opposite direction. The flux lines of the magnetic field compass the wires and are almost trapped in the interior area enclosed by the thin sheet.

Illustration of the shielding effect. We start the series of numerical experiments with direct computations which do not involve the approximate transmission conditions. The numerical computations have been done on meshes $\mathcal{M}^{\varepsilon}$ resolving the sheet (see Fig. 3b) and using curved cells with polynomial degree $p=10$.

In Figure 4, we compare the electromagnetic fields for three different configurations. To each configuration corresponds one column. In the first column no sheet is present, in the second column there is a highly conducting sheet with $\varepsilon=1 / 16, c_{0}=10$, and in the third colum a conducting sheet with $\varepsilon=1 / 16$ and an even higher relative conductivity $c_{0}=100$. The magnetic field is plotted in the first row, whereas the electric field is plotted in the second.

To compare the results the same color scaling is used. In the case of no shielding sheet the fields decays slowly away from the two wires. In the presence of the thin sheets the fields are to some degree trapped in the enclosed area. This is especially pronounced for the most right pictures. The skin depths for the two conducting sheets are $d_{\text {skin }}=0.079=1.26 \varepsilon$ for $c_{0}=10$ and $d_{\text {skin }}=0.025=0.4 \varepsilon$ for $c_{0}=100$.

Numerical study of the modelling error. We compare a numerical approximation of the solution of the models of order 1, 2 and 3 to a reference solution.

For the models of order 1,2 and 3 , the computations were achieved on the mesh $\mathcal{M}^{0}$, see Figure $3 \mathrm{a}$, with no meshing of the thin sheet. We choose $p=18$ for the polynomial degree of the finite element method. The Lagrange multiplier was discretised with discontinuous piecewise polynomial elements on the edges of the interface $\Gamma$.

The reference solutions were computed on the mesh $\mathcal{M}^{\varepsilon}$ which resolves the geometry, see Figure $3 \mathrm{~b}$. The polynomial degree $p=20$ was chosen higher than for the approximate models so that the discretisation error for the reference solution is much smaller than the discretisation error for the model of order 1, 2, 3 and can be neglected.

For the computation of the interior solution we used the Lagrange multiplier for the mean value of the normal derivative and computed locally its second derivative which is present in the model of order 3 . 

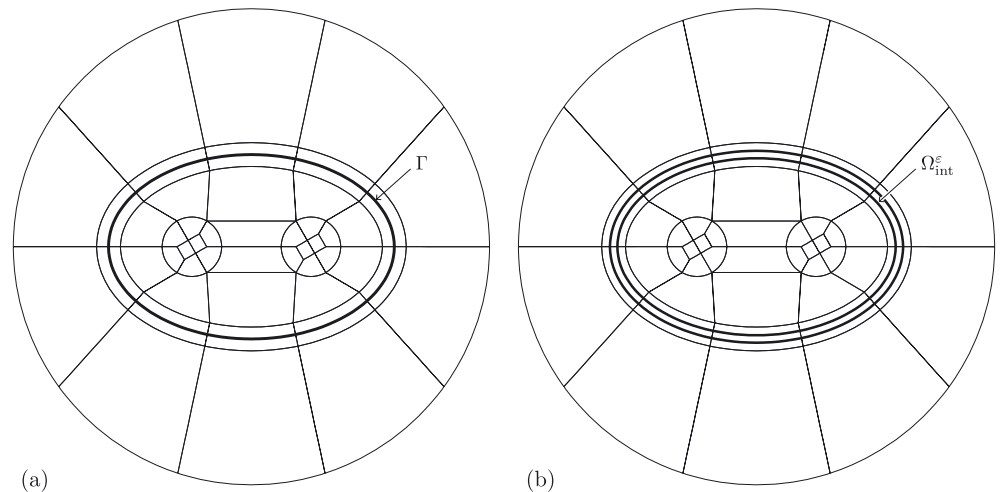

Figure 3. (a) Mesh $\mathcal{M}^{0}$ for the finite element solution of the asymptotic expansion models. The mid-line $\Gamma$ is labelled. (b) Associate mesh $\mathcal{M}^{\varepsilon}$ for the finite element solution of the exact model with the cells in the sheet, here of thickness $\varepsilon=1 / 16$.

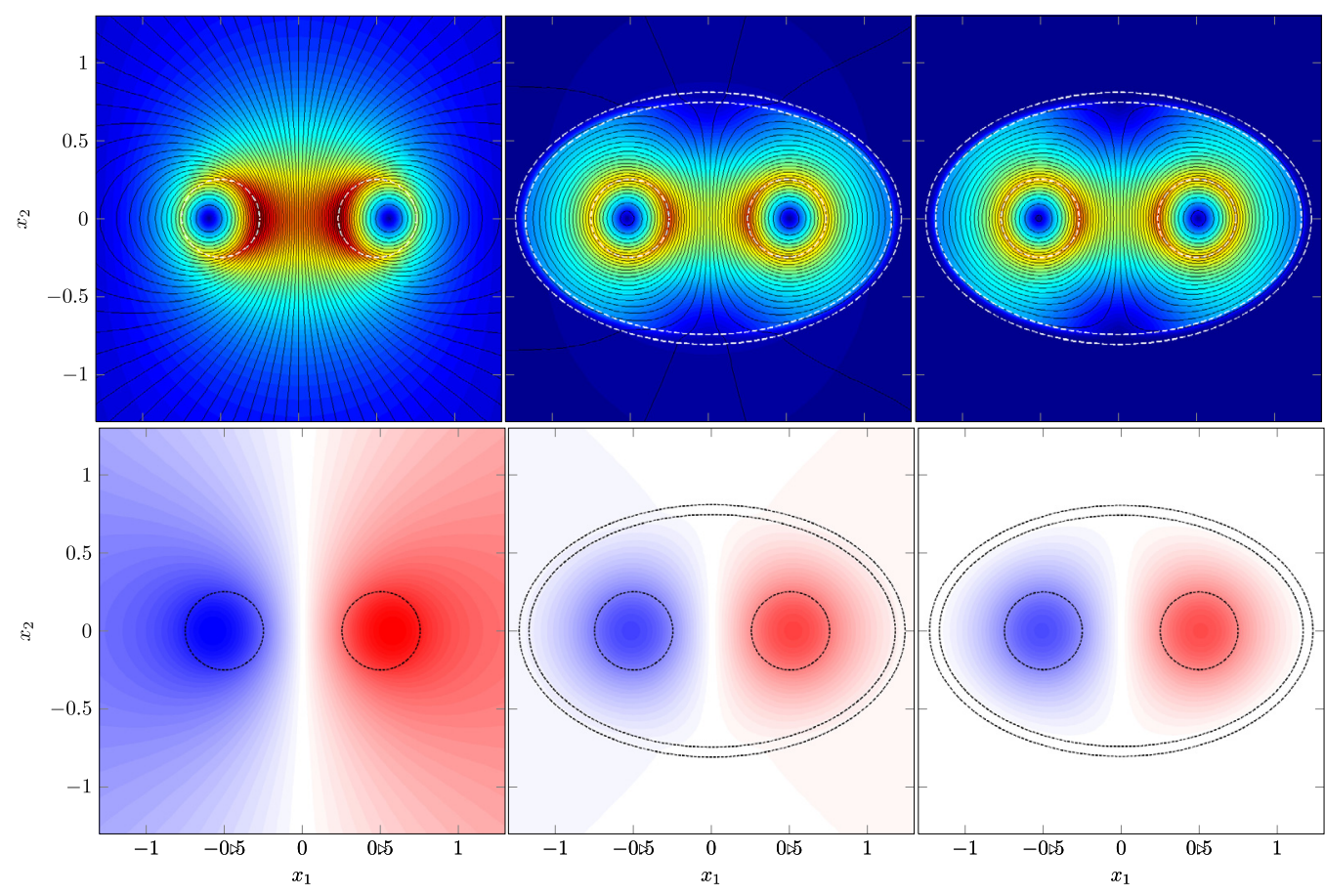

(a) No shielding sheet.

(b) Elliptic sheet with $\varepsilon=1 / 16, c_{0}=10$.

(c) Same sheet with $c_{0}=100$.

FIGURE 4. Illustration of the increasing shielding of two live wires by an elliptic thin sheet of increasing conductivity. Visible is always the same part of the geometric domain. In the top row the magnetic field intensity and direction (flux lines) are shown and in the bottom line the in plane electric field. The scaling of the colours and the distances of the flux lines correspond in all the plots of each row. The parameters are the same as in Figure 2.

Figures 5 and 6 show the convergence of the error of the transmission conditions of order 1,2 and 3 w.r.t. the sheet thickness for the geometry in Figure 2 and relative conductivities $c_{0}=1$ and $c_{0}=250$. The error is shown in $L^{2}$-norm and in $H^{1}$-seminorm in the exterior and the interior of the sheet. 

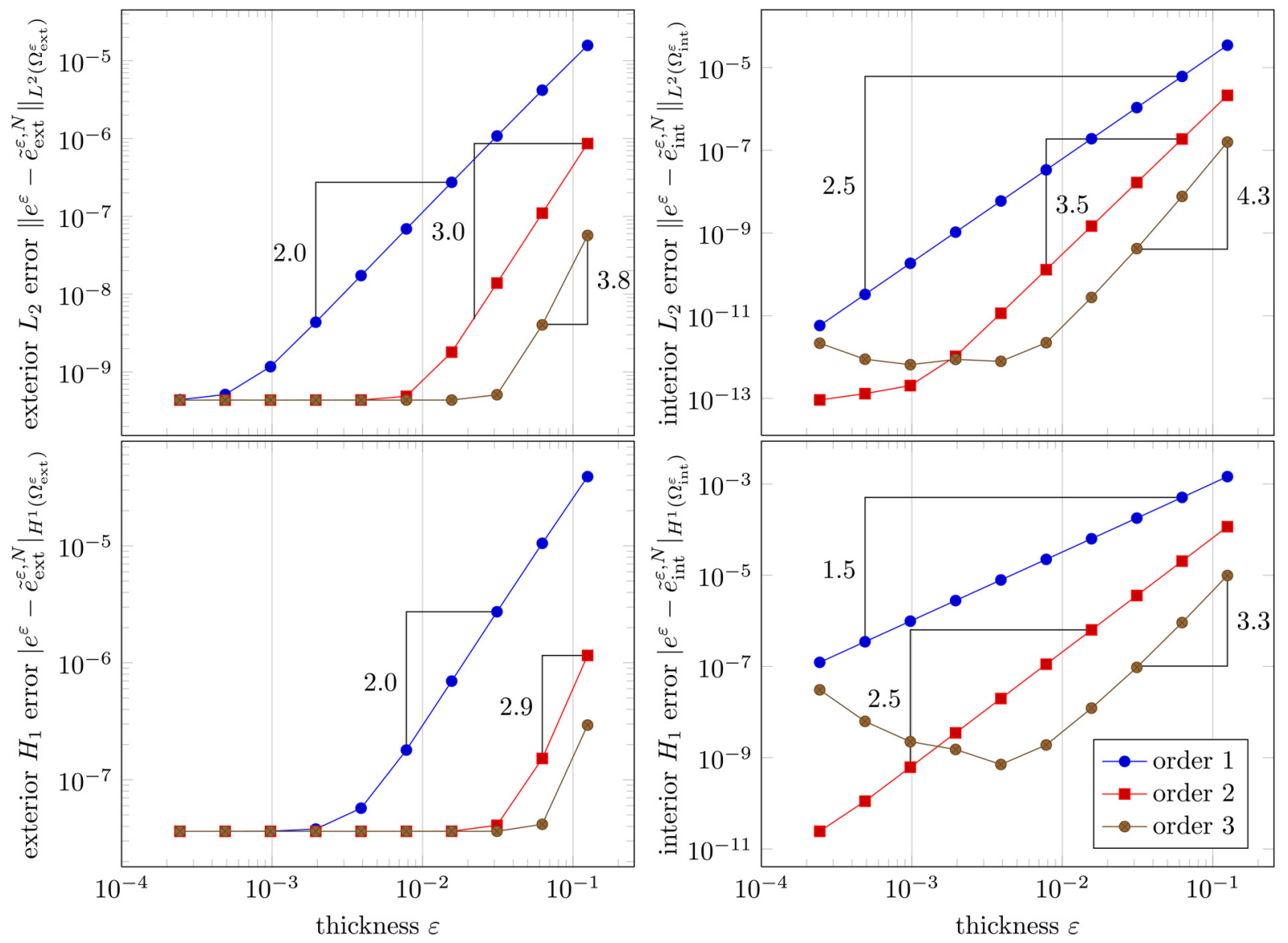

FiguRE 5. Convergence of the error of the transmission conditions of order 1, 2 and 3 for the geometry shown in Figure 2 with $c_{0}=1$ and varying thickness $\varepsilon$. The solution with the transmission condition is computed with $p=18$ and subtracted from a numerical approximation $(p=20)$ to the exact solution to get the error. The error is measured in the $L^{2}\left(\Omega_{\text {ext }}^{\varepsilon}\right)$-norm (top left), in the $L^{2}\left(\Omega_{\text {int }}^{\varepsilon}\right)$-norm (top right), in the $H^{1}\left(\Omega_{\text {ext }}^{\varepsilon}\right)$-seminorm (bottom left), and in the $H^{1}\left(\Omega_{\text {int }}^{\varepsilon}\right)$-seminorm (bottom right). The numerically observed convergence rates verify the estimates in Theorem 4.4, Theorem 5.5 and Remark 3.1. Note, that the $H^{1}$-norm of the exact solution in $\Omega_{\text {ext }}^{\varepsilon}$ is of order 1 , so the given absolute errors corresponds nearly to the relative errors.

For the smallest value of the sheet thickness, the modelling error may fall below the discretisation error. In this case, we observe an horizontal line in the convergence plot.

With $c_{0}=1$ (Fig. 5), we observe very low error levels for all three models and convergence rates which coincide with the theoretically predicted ones. The convergence stops when the discretisation error gets dominant. For the model of order 3 and the error in the interior this point is reached earlier due to inexact evaluation of $\partial_{t}^{2} \tilde{\lambda}_{\text {ext }}^{\varepsilon, 3}=\partial_{t}^{2}\left\{\partial_{n} \tilde{e}_{\text {ext }}^{\varepsilon, 3}\right\}$. For the case $c_{0}=250$ (Fig. 6) we observe almost the same convergence rates as for $c_{0}=1$.

The error distribution of the electric field in the exterior of the sheet is shown in Figure 7 for three examples and the transmission conditions of order 1,2 and 3. The order of magnitude of the error differ largely, and we use therefore for each subfigure its individual colorbars. The first and second examples with sheet thickness $\varepsilon=1 / 16=\frac{0.026}{c_{0}}$ (relative conductivity $\left.c_{0}=10\right)$ and $\varepsilon=1 / 256=\frac{3.91}{c_{0}}$ (relative conductivity $c_{0}=1000$ ) are well and tight, respectively, in the range of validity of the models. We observe also a considerable decrease of the 

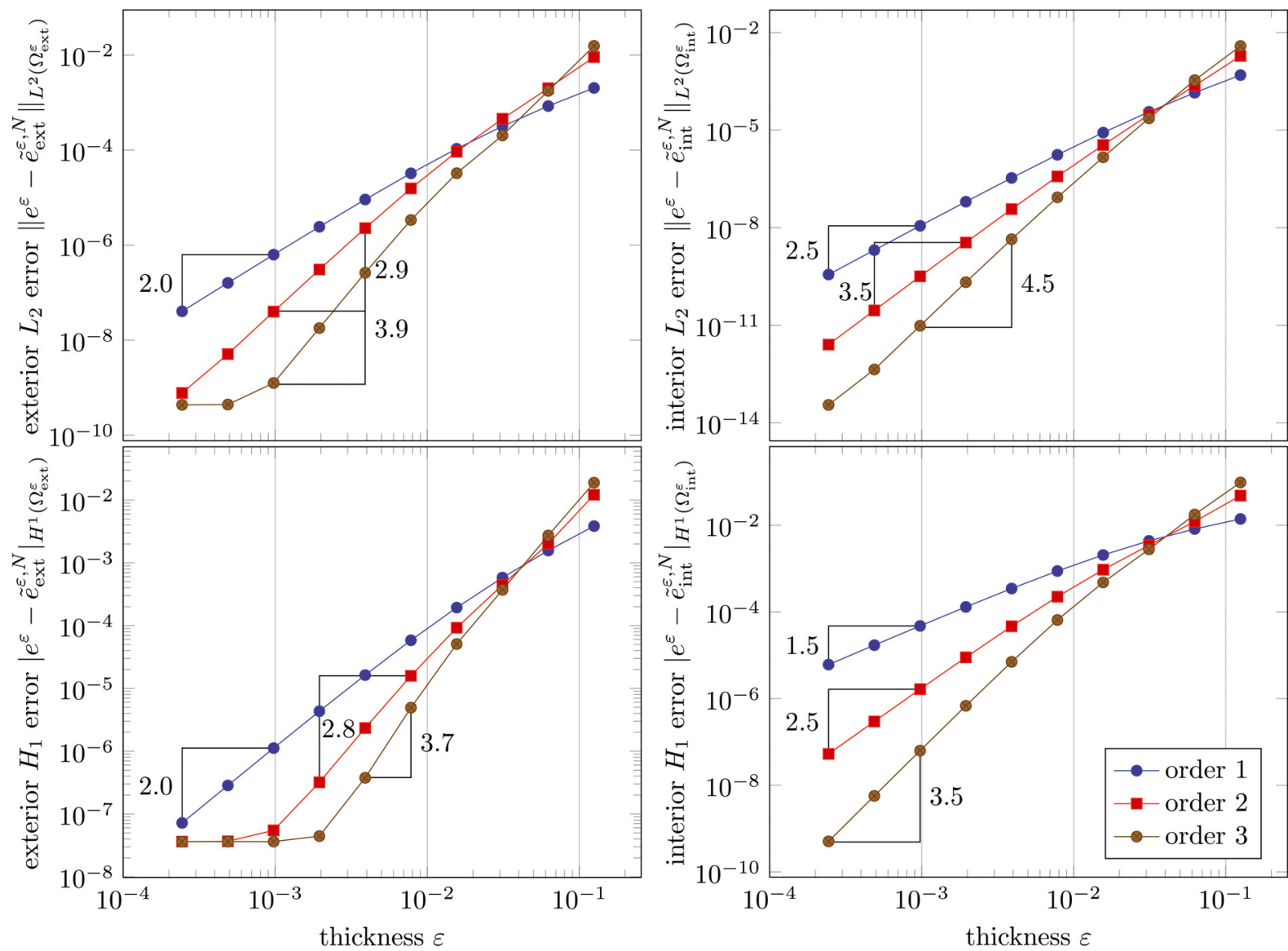

Figure 6. Convergence of the error as in Figure 5, where only the relative conductivity is changed to $c_{0}=250$. The numerically observed rates in $\varepsilon$ correspond again to theoretically predicted. At about $\varepsilon=4 \times 10^{-2}=\frac{10}{c_{0}}$ the curves of different orders have a crossing point $\left(d_{\text {skin }} \approx 3 \varepsilon\right)$. For larger thicknesses $\varepsilon$ the model of order 1 achieves the best results.

error for the first example when increasing the order, whereas the decrease is lesser in the second example. The thickness $\varepsilon=1 / 16=\frac{62.5}{c_{0}}$ (relative conductivity $c_{0}=1000$ ) in the third example lies clearly above the range of validity. The error in this example is smallest for order 1 and increases for higher model orders. In all the examples the error for the order 1 is mainly located in inner area where the electric field itself has highest values. By increasing the model order the error field is more distributed.

For the models of order 2 and 3, the theory presented in the paper is only asymptotic. Lemma 5.2 states for example the stability of the solution only for $\varepsilon$ small enough. During the numerical simulations, no stability problem was observed even for relatively larger values of the factor $c_{0} \varepsilon$ which has reached 31 for $\varepsilon=1 / 8$ and $c_{0}=250$.

\section{Concluding REmarks}

In the context of magneto-quasistatic, we derived three approximate models of order 1,2 and 3 to take into account the far field behaviour of thin and highly conducting sheets. With these models the sheet do not have to be meshed, but is modelled by local transmission conditions. Therefore the models can easily be implemented in most of the finite element libraries or codes based on a Galerkin approximation. Once the field outside the sheet 


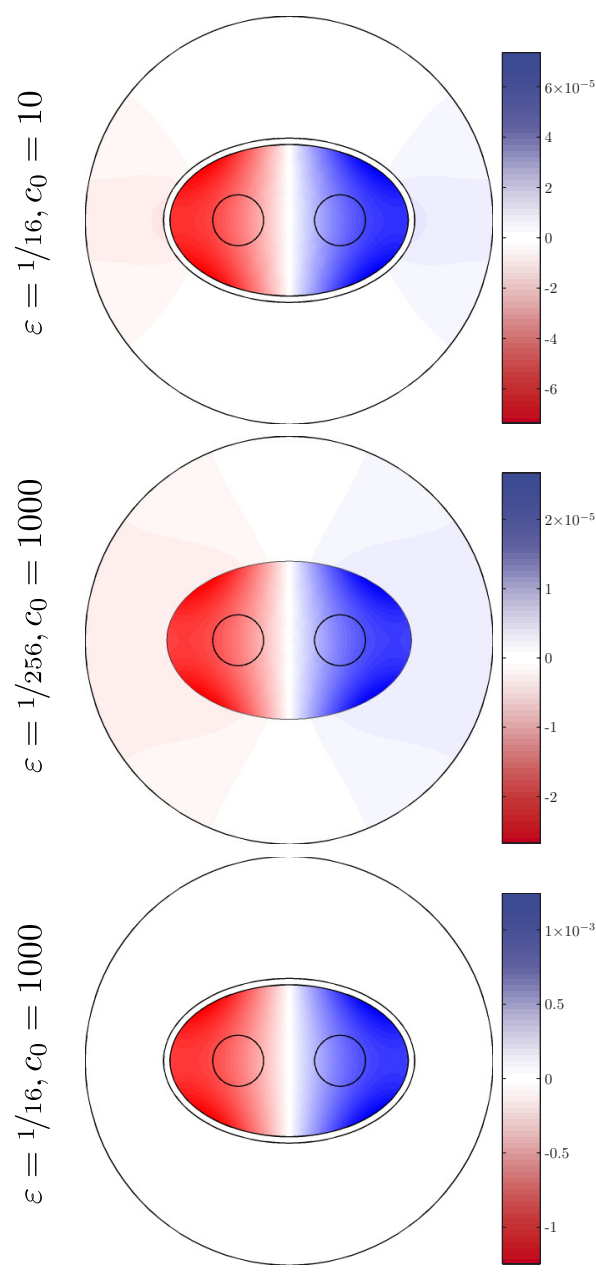

(a) Order 1.
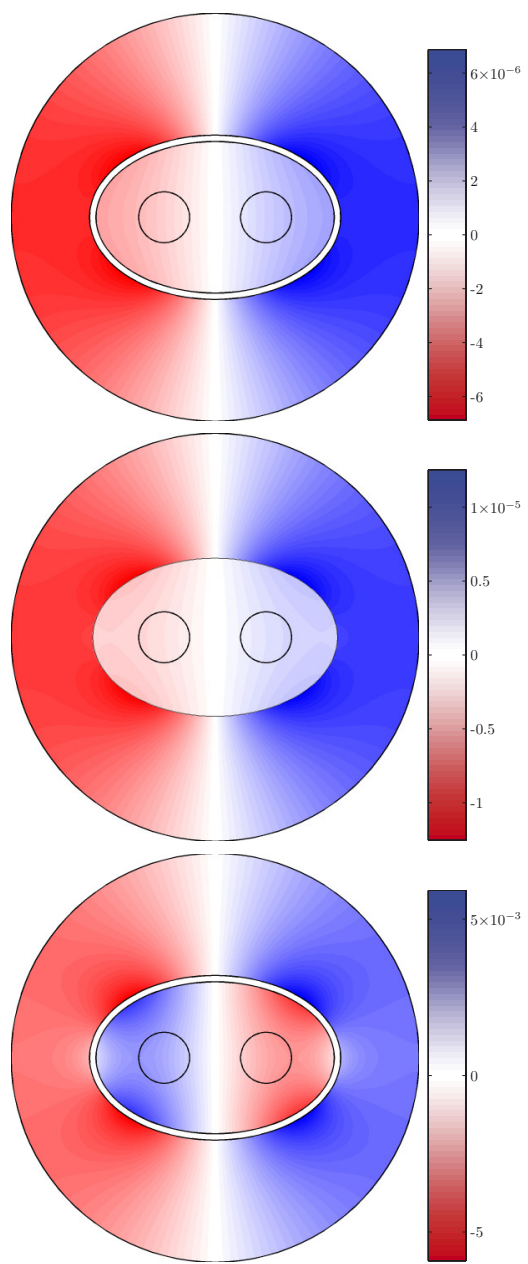

(b) Order 2 .

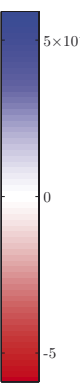

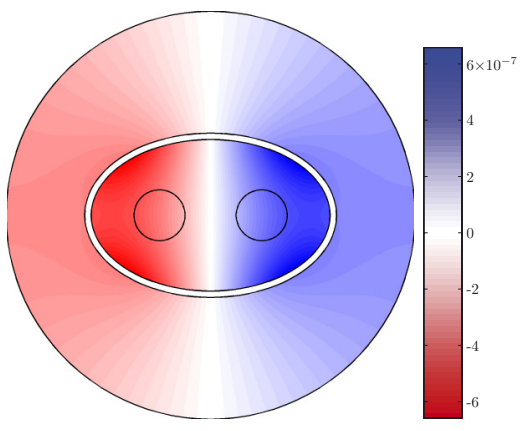
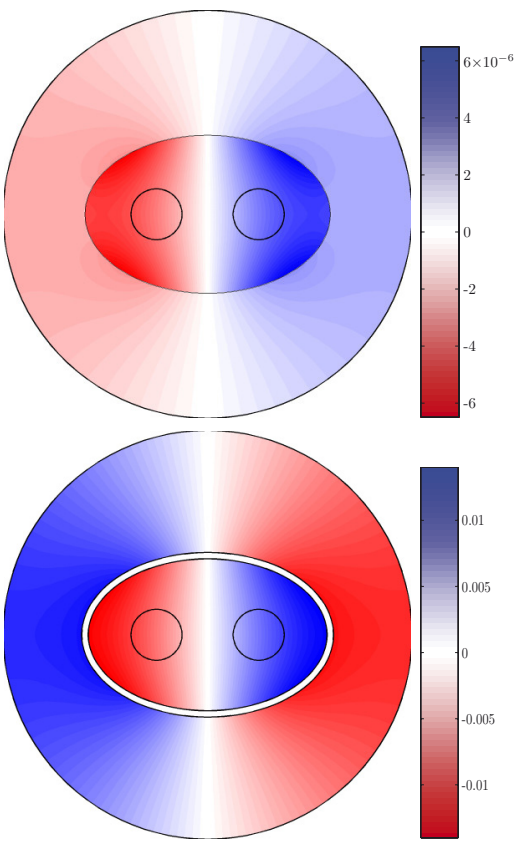

(c) Order 3 .

FIGURE 7. Error of the electric field in the exterior of the thin conducting sheet for the asymptotic models of order 1, 2 and 3 (note the different scalings of the color representation). The configuration in the first row is with a sheet of thickness $\varepsilon=1 / 16$ and relative conductivity $c_{0}=10$. The exact solution for this configuration is shown in Figure $4 \mathrm{~b}$. With this setting the error decreases by about one order of magnitude when increasing the order of the model by one $\left(\varepsilon=\frac{0.625}{c_{0}}\right)$. In the second row the error is plotted for $\varepsilon=1 / 256$ and $c_{0}=1000$ and decreases by a factor of 2 per order $\left(\varepsilon=\frac{3.91}{c_{0}}\right)$. In the third row the configuration is $\varepsilon=1 / 16$ and $c_{0}=1000$ and the error increases with increasing order $\left(\varepsilon=\frac{62.5}{c_{0}}\right)$. Here the skin depth fall below the valid value of the proposed models.

is computed the internal field follows as a polynomial in thickness direction. Our few numerical simulations verify the theoretically achieved estimates for the modelling error. We end this article with some remarks and open problems.

About regularity of the boundary. A lot of industrial casings are polygonal or polyhedral and so contains edges. Our first order approximate model can be applied to such geometries. Anyway its justifications require more advance arguments based on a multiscale analysis similar to the one of [8]. The same type of problematic appears for open sheets. 
About full Maxwell systems in 2D. When the displacement current is not neglected, one has to face a Helmholtz equation inside the exterior domain. This equation has to be supplemented with radiation condition in order to obtain a well posed problem. This leads to a non-coercive variational formulation and the stability results of Sections 4.2 and 5.2 require an important modification (one has to act by contradiction). However, the extra ingredients are now classical. One can refer to [15] for a similar problem.

About three-dimensional thin sheets. When the sheet is not z-invariant but completely three-dimensional, the relevant problem is vectorial. However, in the context of IBC, many authors, see $[4,13]$ for example, have proposed approximate models. One can think to adapt their approach to highly conducting sheets.

\section{A. Appendix}

\section{A.1. The surface operators involved in the transmission conditions}

The approximate solutions $\widetilde{e}_{\mathrm{ext}}^{\varepsilon, N}$ of order $N$ are defined with the help of two differential operators that consists in the truncation of two formal series of operators, see (3.6),

$$
\boldsymbol{\gamma}^{\varepsilon, N}:=\sum_{j=2}^{N} \varepsilon^{j} \boldsymbol{\gamma}_{j} \text { and } \boldsymbol{\zeta}^{\varepsilon, N}:=\sum_{j=1}^{N} \varepsilon^{j} \boldsymbol{\zeta}_{j}
$$

where $\gamma_{0}=\gamma_{1}=\boldsymbol{\zeta}_{0}=0$ and the differential operators $\gamma_{\ell}$ and $\boldsymbol{\zeta}_{\ell}$ that are explicitly given for $\ell \leq 3$ by

$$
\begin{gathered}
\left(\gamma_{2} u\right)(t):=-\frac{\mathrm{i} c_{0}}{24}\left(\kappa(t)\{u\}(t)+2\left\{\partial_{n} u\right\}(t)\right) \\
\left(\gamma_{3} u\right)(t):=-\frac{c_{0}^{2}}{240}\left(\kappa(t)\{u\}(t)+2\left\{\partial_{n} u\right\}(t)\right), \\
\left(\boldsymbol{\zeta}_{1} u\right)(t):=-\frac{c_{0}^{2}}{6}\{u\}(t), \\
\left(\boldsymbol{\zeta}_{2} u\right)(t):=-\frac{\left(\mathrm{i} c_{0}\right)}{12}\left(\frac{7 c_{0}^{2}}{20}+\partial_{t}^{2}\right)\{u\}(t)+\frac{\mathrm{i} c_{0} \kappa(t)}{24}\left\{\partial_{n} u\right\}(t) \\
\left(\boldsymbol{\zeta}_{3} u\right)(t):=\frac{c_{0}^{2}}{40}\left(\frac{17 c_{0}^{2}}{84}+\frac{\kappa^{2}(t)}{3}+\partial_{t}^{2}\right)\{u\}(t)+\frac{c_{0}^{2} \kappa(t)}{240}\left\{\partial_{n} u\right\}(t) .
\end{gathered}
$$

\section{A.2. The interior operators}

The interior approximation of order $N$ involves the differential operators

$$
\boldsymbol{\eta}^{\varepsilon, N}:=\sum_{j=0}^{N} \varepsilon^{j} \boldsymbol{\eta}_{j}
$$


with

$$
\begin{aligned}
\left(\boldsymbol{\eta}_{0} u\right)(t, S)= & \{u\}(t) \\
\left(\boldsymbol{\eta}_{1} u\right)(t, S)= & \frac{\left(\mathrm{i} c_{0}\right)}{2}\{u\}(t)\left(S^{2}+\frac{1}{4}\right)+\left\{\partial_{n} u\right\}(t) S \\
\left(\boldsymbol{\eta}_{2} u\right)(t, S)= & \frac{\left(\mathrm{i} c_{0}\right)^{2}}{24}\{u\}(t)\left(S^{2}+\frac{3}{4}\right)^{2}+\frac{\left(\mathrm{i} c_{0}\right)}{6}\left\{\partial_{n} u\right\}(t)\left(S^{3}-\frac{3}{4} S\right)-\frac{\left(\mathrm{i} c_{0}\right)}{6} \kappa(t)\{u\}(t)\left(S^{3}+\frac{3}{4} S\right) \\
& -\frac{1}{2}\left(\kappa(t)\left\{\partial_{n} u\right\}(t)+\partial_{t}^{2}\{u\}(t)\right) S^{2}, \\
\left(\boldsymbol{\eta}_{3} u\right)(t, S)= & \frac{\left(\mathrm{i} c_{0}\right)^{3}}{720}\{u\}(t)\left(\left(S^{2}+\frac{5}{4}\right)^{3}+\frac{15}{4}\left(S^{2}+\frac{1}{4}\right)\right)+\frac{\left(\mathrm{i} c_{0}\right)^{2}}{120}\left\{\partial_{n} u\right\}(t)\left(S^{2}-\frac{5}{4}\right)^{2} S \\
& +\frac{c_{0}^{2}}{60} \kappa(t)\{u\}(t)\left(S^{4}+\frac{5}{2} S^{2}+\frac{5}{16}\right) S-\frac{\left(\mathrm{i} c_{0}\right)}{12} \kappa(t)\left\{\partial_{n} u\right\}(t)\left(S^{2}-\frac{3}{4}\right) S^{2} \\
& -\frac{\left(\mathrm{i} c_{0}\right)}{12} \partial_{t}^{2}\{u\}(t)\left(S^{2}+\frac{1}{2}\right)\left(S^{2}+\frac{1}{4}\right)+\frac{\left(\mathrm{i} c_{0}\right)}{8} \kappa^{2}(t)\{u\}(t)\left(S^{4}+\frac{1}{2} S^{2}-\frac{1}{48}\right) \\
& +\frac{1}{2}\left(\kappa(t) \partial_{t}^{2}+\frac{1}{3} \kappa^{\prime}(t) \partial_{t}\right)\{u\}(t) S^{3}+\frac{1}{3}\left(\kappa^{2}(t)-\frac{1}{2} \partial_{t}^{2}\right)\left\{\partial_{n} u\right\}(t) S^{3} .
\end{aligned}
$$

\section{B. Existence, uniqueness And REGUlarity of THE TERMS}

\section{OF THE ASYMPTOTIC EXPANSIONS}

We recall that $\Omega$ is a connected bounded domain of $\mathbb{R}^{2}$ with regular boundary $\partial \Omega$ and that $\Gamma$ is a closed regular curve included in $\Omega$. The two connected components of $\Omega$ are denoted by $\Omega_{+}$and $\Omega_{-}$, see Figure 1 .

In this appendix, we aim in proving that the terms of the asymptotic expansions are well-defined and regular.

Lemma B.1. Let $f \in C^{\infty}(\bar{\Omega}), g \in C^{\infty}(\Gamma)$, and $h \in C^{\infty}(\Gamma)$. There exists a unique $u \in H^{1}(\Omega \backslash \Gamma)$

$$
\begin{aligned}
-\Delta u(\underline{x}) & =f(\underline{x}), & & \text { in } \Omega \backslash \Gamma, \\
{[u](t) } & =g(t), & & \text { on } \Gamma, \\
{\left[\partial_{n} u\right](t)-\mathrm{i} c_{0}\{u\}(t) } & =h(t), & & \text { on } \Gamma, \\
u & =0, & & \text { on } \partial \Omega .
\end{aligned}
$$

Moreover, $u$ is regular in $\Omega_{+}$and $\Omega_{-}$, i.e., $u \in C^{\infty}\left(\bar{\Omega}_{+}\right)$and $u \in C^{\infty}\left(\bar{\Omega}_{-}\right)$.

Proof. The proof consists of two parts, the proof of existence and uniqueness, and the proof of the regularity.

(i) Existence and uniqueness. Since $[u](t)=g(t)$, the function $u$ is in general not in $H^{1}(\Omega)$. We introduce the auxiliary function $\hat{u}$ defined by

$$
\hat{u}(\underline{x})=u(\underline{x})-\chi(\underline{x}) w(\underline{x})
$$

with

- $w \in H^{1}\left(\mathbb{R}^{2} \backslash \Gamma\right)$ satisfying

$$
\begin{aligned}
-\Delta w(\underline{x}) & =f(\underline{x}), \quad \text { in } \mathbb{R}^{2} \backslash \Gamma, \\
{[w](t) } & =g(t), \quad \text { on } \Gamma, \\
{\left[\partial_{n} w\right](t) } & =0, \quad \text { on } \Gamma,
\end{aligned}
$$


and which is defined by the representation formula

$$
w(\underline{x})=\int_{\Omega} G\left(\underline{x}, \underline{x}^{\prime}\right) f\left(\underline{x}^{\prime}\right) \mathrm{d} \underline{x}^{\prime}+\int_{\Gamma} \frac{\partial G}{\partial n}\left(\underline{x}, \underline{x}^{\prime}\right) g\left(\underline{x}^{\prime}\right) \mathrm{d} \underline{x}^{\prime}
$$

with $G$ the Green function of the operator $-\Delta$ in $2 \mathrm{D}$

$$
G\left(\underline{x}, \underline{x}^{\prime}\right)=-\frac{1}{2 \pi} \ln \left\|\underline{x}-\underline{x}^{\prime}\right\|
$$

- the function $\chi$ a regular cut-off function vanishing in a neighbourhood of $\partial \Omega$ and with value 1 in a neighbourhood of $\Gamma$.

The function $\hat{u} \in H_{\partial \Omega}^{1}(\Omega)$ satisfies

$$
\begin{aligned}
-\Delta \hat{u}(\underline{x}) & =\hat{f}(\underline{x}), \quad \text { in } \Omega, \\
{\left[\partial_{n} \hat{u}\right](t)-\mathrm{i} c_{0} \hat{u}(t) } & =\hat{h}(t), \quad \text { on } \Gamma,
\end{aligned}
$$

with $\hat{f}=f+\Delta(\chi w) \in C^{\infty}(\Omega)$ (this function vanishes in the neighbourhood of $\Gamma$ ) and $\hat{h}=h+\mathrm{i} c_{0}\{w\} \in H^{1 / 2}(\Gamma)$. This strong formulation is equivalent to the well-posed variational formulation (due to the Lax-Milgram theorem and Poincaré-Friedrich inequality) seek $\hat{u} \in H_{\partial \Omega}^{1}(\Omega)$ such that

$$
\int_{\Omega} \nabla \hat{u} \cdot \nabla \overline{u^{\prime}} \mathrm{d} \underline{x}+\int_{\Gamma} \mathrm{i} c_{0} \hat{u} \overline{u^{\prime}} \mathrm{d} t=\int_{\Omega} \hat{f} \overline{u^{\prime}} \mathrm{d} \underline{x}-\int_{\Gamma} \hat{h} \overline{u^{\prime}} \mathrm{d} t, \quad \forall u^{\prime} \in H_{\partial \Omega}^{1}(\Omega) .
$$

This demonstrates that $u \in H_{\partial \Omega}^{1}(\Omega \backslash \Gamma)$ is uniquely defined by (B.1).

(ii) Regularity. To show that the function $u \in C^{\infty}\left(\bar{\Omega}_{+}\right) \times C^{\infty}\left(\bar{\Omega}_{-}\right)$we use a key argument coming from the integral representation theory [18]. Let $s \geq 1$. Since $\partial \Omega$ and $\Gamma$ are regular, we have

$$
\left.\begin{array}{c}
u \in H^{1}\left(\Omega_{-}\right) \text {and } u \in H^{1}\left(\Omega_{+}\right) \text {and } u=0 \text { on } \partial \Omega, \\
\Delta u \in H^{s-2}\left(\Omega_{-}\right) \text {and } \Delta u \in H^{s-2}\left(\Omega_{+}\right) \\
{[u] \in H^{s-\frac{1}{2}}(\Gamma) \text { and }\left[\partial_{n} u\right] \in H^{s-\frac{3}{2}}(\Gamma)}
\end{array}\right\} \quad \Longrightarrow \quad u \in H^{s}\left(\Omega_{+}\right) \text {and } u \in H^{s}\left(\Omega_{-}\right) \text {, }
$$

or equivalently

$$
\left.\begin{array}{c}
u \in H_{\partial \Omega}^{1}(\Omega \backslash \Gamma) \text { and } \Delta u \in H^{s-2}(\Omega \backslash \Gamma) \\
{[u] \in H^{s-\frac{1}{2}}(\Gamma) \text { and }\left[\partial_{n} u\right] \in H^{s-\frac{3}{2}}(\Gamma)}
\end{array}\right\} \quad \Longrightarrow \quad u \in H^{s}(\Omega \backslash \Gamma)
$$

We act by induction. Let us suppose that $u$ belongs to $H^{m}(\Omega \backslash \Gamma)$ and prove that $u \in H^{m+1}(\Omega \backslash \Gamma)$ with $m \geq 1$. By trace theorem $\{u\} \in H^{m-\frac{1}{2}}(\Gamma)$. Consequently, this leads to

$$
\left.\begin{array}{c}
u \in H_{\partial \Omega}^{1}(\Omega \backslash \Gamma) \text { and } \Delta u \in C^{\infty}\left(\bar{\Omega}_{+}\right) \times C^{\infty}\left(\bar{\Omega}_{-}\right) \\
u]=g \in C^{\infty}(\Gamma) \text { and }\left[\partial_{n} u\right]=\mathrm{i} c_{0}\{u\}+h \in H^{m-\frac{1}{2}}(\Gamma)
\end{array}\right\} \quad \Longrightarrow \quad u \in H^{m+1}(\Omega \backslash \Gamma) \text {. }
$$

This ends the proof.

\section{REFERENCES}

[1] X. Antoine, H. Barucq and L. Vernhet, High-frequency asymptotic analysis of a dissipative transmission problem resulting in generalized impedance boundary conditions. Asymptot. Anal. 26 (2001) 257-283.

[2] N. Bartoli and A. Bendali, Robust and high-order effective boundary conditions for perfectly conducting scatterers coated by a thin dielectric layer. IMA J. Appl. Math. 67 (2002) 479-508. 
[3] A. Bendali and K. Lemrabet, The effect of a thin coating on the scattering of a time-harmonic wave for the Helmholtz equation. SIAM J. Appl. Math. 6 (1996) 1664-1693.

[4] A. Bendali and K. Lemrabet, Asymptotic analysis of the scattering of a time-harmonic electromagnetic wave by a perfectly conducting metal coated with a thin dielectric shell. Asymptot. Anal. 57 (2008) 199-227.

[5] A. Bossavit, Computational Electromagnetism. Variational Formulation, Complementarity, Edge Elements. No. 2 in Academic Press Electromagnetism Series. Academic Press, San Diego (1998).

[6] D. Braess, Finite Elements: Theory, Fast Solvers, and Applications in Solid Mechanics, 3th edition. Cambridge University Press (2007).

[7] H. Brezis, Functional Analysis, Sobolev Spaces and Partial Differential Equations. Springer-Verlag, New York (2010).

[8] G. Caloz, M. Costabel, M. Dauge and G. Vial, Asymptotic expansion of the solution of an interface problem in a polygonal domain with thin layer. Asymptot. Anal. 50 (2006) 121-173.

[9] Concepts Development Team. Webpage of Numerical C++ Library Concepts 2. http://www.concepts.math.ethz.ch (2011).

[10] M. Duruflé, H. Haddar and P. Joly, Higher order generalized impedance boundary conditions in electromagnetic scattering problems. C.R. Phys. 7 (2006) 533-542.

[11] P. Frauenfelder and C. Lage, Concepts - an object-oriented software package for partial differential equations. ESAIM: M2AN 36 (2002) 937-951.

[12] H. Haddar, P. Joly and H.M. Nguyen, Generalized impedance boundary conditions for scattering by strongly absorbing obstacles: the scalar case. Math. Models Methods Appl. Sci. 15 (2005) 1273-1300.

[13] H. Haddar, P. Joly and H.M. Nguyen, Generalized impedance boundary conditions for scattering by strongly absorbing obstacles: the case of Maxwell s equations. Math. Models Methods Appl. Sci. 18 (2008) 1787-1827.

[14] H. Igarashi, A. Kost and T. Honma, A boundary element analysis of magnetic shielding for electron microscopes. Compel 17 (1998) 585-594.

[15] P. Joly and S. Tordeux, Asymptotic analysis of an approximate model for time harmonic waves in media with thin slots. ESAIM: M2AN 40 (2006) 63-97.

[16] L. Krähenbühl and D. Muller, Thin layers in electrial engineering. Example of shell models in analysing eddy-currents by boundary and finite element methods. IEEE Trans. Magn. 29 (1993) 1450-1455.

[17] M.A. Leontovich, On approximate boundary conditions for electromagnetic fields on the surface of highly conducting bodies (in russian). Research in the propagation of radio waves. Moscow, Academy of Sciences (1948) 5-12.

[18] W. McLean, Strongly Elliptic Systems and Boundary Integral Equations. Cambridge University Press (2000).

[19] A.M. Miri, N.A. Riegel and C. Meinecke, FE calculation of transient eddy currents in thin conductive sheets using dynamic boundary conditions. Int. J. Numer. Model. 11 (1998) 307-316.

[20] T. Nakata, N. Takahashi, K. Fujiwara and Y. Shiraki, 3D magnetic field analysis using special elements. IEEE Trans. Magn. 26 (1990) 2379-2381.

[21] V. Péron and C. Poignard, Approximate transmission conditions for time-harmonic Maxwell equations in a domain with thin layer. Research Report RR-6775, INRIA (2008).

[22] R. Perrussel and C. Poignard, Asymptotic Transmission Conditions for Steady-State Potential in a High Contrast Medium. A Uniform Variational Formulation for Resistive Thin Layers. Research Report RR-7163, INRIA (2010).

[23] K. Schmidt, High-order numerical modeling of highly conductive thin sheets. Ph.D. thesis, ETH Zürich (2008).

[24] K. Schmidt and S. Tordeux, Asymptotic modelling of conductive thin sheets. Z. Angew. Math. Phys. 61 (2010) $603-626$.

[25] K. Schmidt, O. Sterz and R. Hiptmair, Estimating the eddy-current modelling error. IEEE Trans. Magn. 44 (2008) 686-689.

[26] T. Senior and J. Volakis, Approximate Boundary Conditions in Electromagnetics. Institution of Electrical Engineers (1995).

[27] A.N. Shchukin, Propagation of Radio Waves (in Russian). Svyazizdat, Moscow (1940). 